\title{
Single-nucleon knockout cross sections for reactions producing resonance states at or beyond the drip line
}

\author{
R. J. Charity ${ }^{1}$ and L. G. Sobotka ${ }^{1,2}$ \\ ${ }^{1}$ Department of Chemistry, Washington University, St. Louis, MO 63130, USA and \\ ${ }^{2}$ Department of Physics, Washington University, St. Louis, MO 63130, USA \\ J. A. Tostevin \\ Department of Physics, Faculty of Engineering and Physical Sciences, \\ University of Surrey, Guildford, Surrey GU2 7XH, United Kingdom
}

\begin{abstract}
Single-nucleon knockout cross sections from fast secondary beams of the proton-drip-line nuclei ${ }^{9} \mathrm{C},{ }^{13} \mathrm{O}$, and ${ }^{17} \mathrm{Ne}$ on a ${ }^{9} \mathrm{Be}$ target have been studied with emphasis on the production of resonance states. These states were identified by their invariant mass and resonances with two, three, and fivebody exit channels were examined. The measured cross sections for these states were compared to eikonal-model predictions using shell-model or Variational Monte Carlo spectroscopic factors. The experimental yields were found to be suppressed relative to the model predictions, especially when a well-bound neutron or proton is removed. This suppression exceeds that found systematically in measured inclusive cross sections to particle-bound final states. In neutron knockout from ${ }^{9} \mathrm{C}$ and ${ }^{13} \mathrm{O}$ projectiles this suppression of the unbound ground-state residuals yield is a factor of $2-3$ times larger than that found in the bound final-state studies. Modifications to the structure of these systems due to coupling of the shell-model configurations to the continuum is expected to contribute to this extra suppression, especially when the final state is a near-threshold resonance. However, other considerations including the role of nuclear dynamics may be required to explain all the observed trends.
\end{abstract}

\section{INTRODUCTION}

Nucleon knockout reactions from fast secondary beams on light target nuclei such as ${ }^{9} \mathrm{Be}$ and ${ }^{12} \mathrm{C}$ have proven an effective way of making some of the most exotic isotopes far removed from the line of $\beta$ stability. A much-used theoretical model of such single-nucleon knockout reactions makes use of the sudden and eikonal approximations [1]. The theoretical cross section to a given shell-model final state, that combines structure and dynamical information, is

$$
\sigma_{t h}=\left(\frac{A}{A-1}\right)^{N} C^{2} S \sigma_{s p} .
$$

Here, the first term is a center-of-mass correction (for projectile mass $A$ ) to the shell-model spectroscopic factor $C^{2} S$ for the overlap of the initial and final states and $N$ is the number of oscillator quanta of the major shell of the removed particle. The single-particle cross section, $\sigma_{s p}$, calculated assuming a single-particle overlap normalized to unity, is the sum of contributions from elastic and inelastic knockout processes [1].

A systematic comparison of the inclusive, bound-finalstates cross sections, $\sigma_{i n c}$, from this theoretical approach, obtained by summing the individual $\sigma_{t h}$ for all shellmodel final states with excitation energies below the first nucleon threshold, to the measured inclusive cross sections to bound residuals, $\sigma_{\text {exp }}$ was performed in Ref. [2] and subsequently updated in Ref. [3]. The observed cross sections ratio, $R_{s}=\sigma_{\text {exp }} / \sigma_{i n c}$, as a function of $\Delta S$, the difference in separation energies of the two nucleon species from the projectile ground state, is reproduced in Fig. 1. Specifically, $\Delta S=S_{p}-S_{n}$ for proton removal reactions and $S_{n}-S_{p}$ for neutron removal reactions, $\Delta S$ being large and positive in cases where one removes a strongly-bound nucleon. It was found that, within an error band of order $0.1, R_{s}$ falls essentially linearly from around unity in reactions with large negative $\Delta S$ to values of typically $0.3(1)$ for the largest measured $\Delta S$ of $\approx 15-20 \mathrm{MeV}$. The latter arise when the removal is of a nucleon of the minority species from near its well-bound Fermi-surface in a highly $(N, Z)$ asymmetric nucleus. In this work we refer to these trends in the $R_{s}$ behavior as the bound-states or $R_{s}$ systematics. These systematic studies were prompted when such an unexpectedly small $R_{s}=0.24(3)$ was found in neutron knockout from the proton-rich ${ }^{32} \mathrm{Ar}$ projectile, having $\Delta S \approx 20 \mathrm{MeV}$ [4]. In that case, and some other systems furthest from stability, the only bound final state was the ground-state of the residual.

The origin of the systematic behavior of $R_{s}$ within the eikonal-model analyses is unresolved at present but may originate from missing physics or from inadequacies in the reaction dynamical (e.g. the sudden approximation) and/or the nuclear-structure model. In particular, the role and treatment of correlations in the many-body wave functions of (i) near-continuum systems, and (ii) of $n p$ correlations at the energetically well-bound Fermisurface of the minority species, embedded as it is, spatially, within nuclear matter comprised of the majority nucleon species. A recent, completely different model approach [5] applied to inclusive cross sections for fast neutron and proton removal reactions from neutron-rich medium-mass nuclei, i.e. for reactions with larger negative and positive $\Delta S$ values, also calculated asymmetric $R_{s}$ values with calculated yields significantly exceeding measured values for the proton removal cases. There it was argued that short-range correlations are respon- 


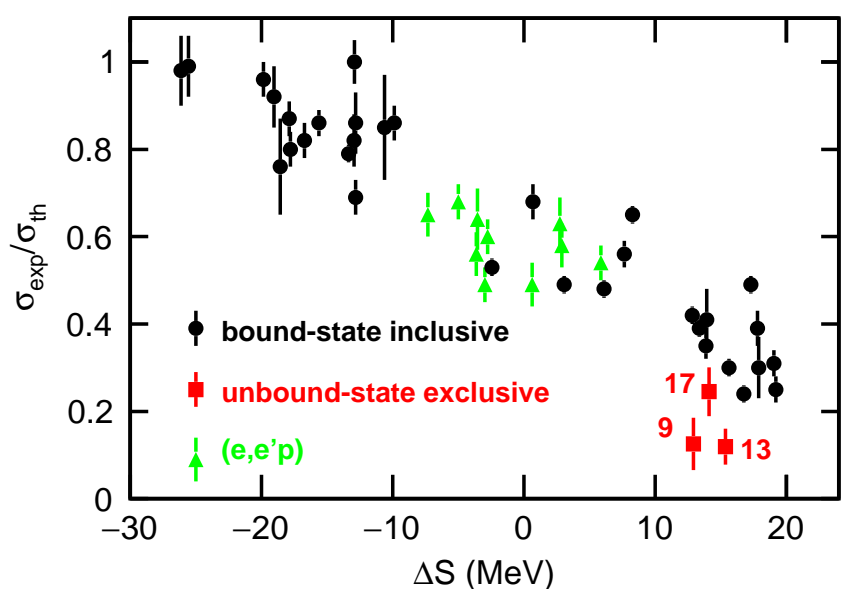

FIG. 1. The systematic behavior of the ratio of experimental to theoretical inclusive neutron and proton knockout cross sections to particle-bound residuals, from Tostevin and Gade [3] (circular data points) plotted as a function of $\Delta S$, the difference in the separation energies of the two nucleon species (see text). Also shown are the $R_{u}$ values, the cross section ratios determined from exclusive neutron knockout cross sections to particle-unbound states, obtained in this work (red squares). For the latter, the points are labeled by the massnumber of the projectile. Also shown are the ratios obtained for $\left(e, e^{\prime} p\right)$ reactions on stable isotopes, taken from [3].

sible. One possibility is that the physical spectroscopic factors are suppressed compared to the (truncated model space) shell-model predictions for removal of the minority nucleon species. In attempts to probe this, related cross section ratios, for more limited reaction sets and nuclear species, were also generated using nucleon-removal by transfer $[6-8]$ and quasi-free $(p, 2 p)$ knockout $[9,10]$ processes. However, in the case of the transfer reactions, the ratios were generally computed from selected exclusive (e.g. ground-state) cross sections and not boundfinal-state inclusive yields while the $(p, 2 p)$ analysis was restricted to proton removal from oxygen isotopes. However, more constant ratios as a function of $\Delta S$ were obtained in these studies.

The discussion above relates only to bound final states. To date, there is a paucity of cross section data on singlenucleon removal reactions leading to resonances. Nucleon removal from projectiles located at the drip line is often used as an effective way to create and study the ground and excited states of particle-unstable states beyond the drip line. Most often such studies do not measure absolute cross sections. However, in planning experiments, quantitative cross section estimates are needed for reliable beam-time requests. Thus, whether the eikonal model and observed bound-states inclusive yield systematics are applicable also for the exclusive yields of resonances is of interest. Such cross sections for resonances might also help elucidate the physics behind the observed $\Delta S$ dependence seen in $R_{s}$. However other factors may be important for resonances. For example, the applicability of the standard (harmonic-oscillator basis) shell- model, which treats the nucleus as a closed system may have limitations. The study of resonance production cross sections near the drip lines is thus important to help quantify the theoretical models presently used.

In this work we report on neutron and proton knockout cross sections from light projectiles located at the proton drip line. The experimental data, using the invariantmass technique to study the structure and decay properties of resonances in exotic nuclei, come from previously published works. These experiments were not optimized to determine knockout cross sections, but nevertheless provide useful results. In particular, we will present cross sections for ${ }^{9} \mathrm{C},{ }^{13} \mathrm{O}$, and ${ }^{17} \mathrm{Ne}$ projectiles. The particle-unstable states produced following one nucleon removal were observed to sequentially decay into two, three, or five-body exit channels. For determining the cross sections, the challenge is to understand the detector response so that the detection efficiencies can be accurately determined. In order to identify a particular residual level in the invariant-mass method, all of its decay products must be detected, which can lead to small efficiencies for the exit channels with larger multiplicities. Statistical uncertainties are minimal in most, but not all of the deduced cross sections, and the uncertainties presented are systematic unless otherwise specified.

\section{HIRA DATA AT $E / A \approx 60 \mathrm{MEV}$}

With one exception, the data presented in this study where obtained using fast secondary beams produced at the National Superconducting Cyclotron Laboratory at Michigan State University. In all cases, the secondary beams were incident on the same 1 -mm-thickness ${ }^{9} \mathrm{Be}$ target and the decay products were detected in the High Resolution Array (HiRA) [11] with nearly identical configurations covering the same angular regions of the decay products $\left(\approx 2^{\circ}-12^{\circ}\right)$. In these experiments, HiRA consisted of $14 \mathrm{Si}-\mathrm{CsI}(\mathrm{Tl}) E-\Delta E$ telescopes. Each telescope contained a double-side Si strip detector which provided position (scattering angle) information and behind this was placed a $2 \times 2$ array of $\mathrm{CsI}(\mathrm{Tl})$ crystals. This arrangement allowed for multi-hits in the same telescope to be identified and thus increased the detection efficiency. The particle-unstable residuals from the knockout reaction were isolated from the invariant mass of their decay products.

The multi-particle detection efficiencies were calculated using Monte Carlo simulations [12] where most of the detection loss is due to the geometric acceptance of the decay products, which must be detected in coincidence. To determine the geometric contribution accurately, the relative positions and orientations of each HiRA telescope were measured with an accuracy of $28 \mu \mathrm{m}$ with a coordinate machine arm. The latter, also called a ROMER arm, is a portable mechanical arm that precisely measures an object in a $3 \mathrm{D}$ coordinate system. The next most important ingredient is the angular distributions of the parent residual fragments after the knockout 
reaction. These must be determined for each residual state and are discussed in detail later. The residual fragments are assumed to sequentially decay isotropically. We have checked that reconstructed angular distributions of the decay fragments in the parent frame are consistent between the experiment and the "detected" simulated events, indicating that any spin-alignment of the higher spin states is small in the cases studied.

The 3-body exit channels observed in this work correspond to prompt $2 p$ decay and correlations between the momenta of the decay fragments must be included in the simulations. These were initially taken from the experimental-measured correlations (Jacobi plots). Some examples for states considered in this work can be found in Refs. [13-15]). The Monte Carlo simulations show that the effect of the experimental resolution is small and a simple iterative technique was used to correct the experimental distributions for this resolution. For some states, theoretical correlations are also available which are consistent with these corrected distributions $[14,15]$. The most uncertain case studied is the 5-body decay of ${ }^{8} \mathrm{C}$ which consists of two sequential steps of $2 p$ decay [16]. The second step involves the decay of ${ }^{6} \mathrm{Be}^{g . s}$. for which the correlations have been measured accurately [17]. However, the correlations for the first step are more uncertain. Some information on the first-step correlations was obtained in Ref. [16], but to estimate the uncertainty we also tried two other variants for the correlations; randomly sampling the three-body phase space and assuming more diproton-like correlations.

Some loss of events also occurs if a particle enters the region where two $\mathrm{CsI}(\mathrm{Tl})$ crystals from the same telescope adjoin (Note, there are $4 \mathrm{CsI}(\mathrm{Tl})$ crystals in each telescope). Here the particle can enter one $\mathrm{CsI}(\mathrm{Tl})$ crystal, pass through the separating material and even stop in an adjacent crystal so it will not fall within its $E-\Delta E$ gate and be identified. To determine these losses, we have scanned the yield for each detected particle type as a function of Si strip position, on both sides of the Si detector, and observed dips in the yield where the crystals adjoin. The magnitude of these dips is then used in the Monte Carlo simulations to incorporate this effect. For example, with three decay products, this gave a total reduction of $\approx 25 \%$ in the efficiency.

A second correction is made for fragments which pass into a single $\mathrm{CsI}(\mathrm{Tl})$ crystal but still do not lie in their $E-$ $\Delta E$ gate due to processes including nuclear reactions in the $\mathrm{CsI}(\mathrm{Tl})$ material $[18,19]$, incomplete charge collection in the Si detectors due to crystal defects, and channeling in the Si crystals. This correction is determined from the calibration beams used to obtain the particle-dependent energy calibration for the $\mathrm{CsI}(\mathrm{Tl})$ detectors. However, with these beams we also determined the fraction of the yield in the $E-\Delta E$ plots that lies outside of the employed particle-identification gates. For three decay products, we lose $\sim 18 \%$ of the events from these processes.

\section{A. knockout from ${ }^{9} \mathrm{C}$}

Neutron and proton knockout from ${ }^{9} \mathrm{C}$ projectiles is studied using data from two HiRA experiments at $E / A \approx 66.8 \mathrm{MeV}$. In addition to knockout to resonant states, we also look at proton knockout to the ground state of ${ }^{8} \mathrm{~B}$, which is particle stable. As ${ }^{8} \mathrm{~B}$ has no particle-stable excited states to feed the ground-state by $\gamma$ decay, this measured cross section will also be exclusive. In the first experiment $[16,20]$, the gains for the Si amplifiers were set up for $p$ and $\alpha$ detection, appropriate to detect the $4 p+\alpha$ exit channel of ${ }^{8} \mathrm{C}^{g \cdot s}$ produced following neutron knockout from the projectile. The proton knockout channels were not identified as the heavier decay products saturated the Si amplifiers. However, in a second experiment [21], with almost exactly the same arrangement of the HiRA detectors, lower-gain amplifiers were used allowing the detection of the $p+{ }^{7} \mathrm{Be}$ and $2 p+{ }^{6} \mathrm{Li}$ decay channels from excited ${ }^{8} \mathrm{~B}$ states. In addition, the gains for the two inner-most detectors were appropriate to determine the inclusive ${ }^{8} \mathrm{~B}$ ground-state yield over a more limited ranges of angles. In this second experiment, a few of the $\mathrm{CsI}(\mathrm{Tl})$ detectors did not function which significantly reduced the ${ }^{8} \mathrm{C} \rightarrow 4 p+\alpha$ detection efficiency. We therefore use the first experiment for the neutron knockout channel and the second for the protonknockout states. The accumulative number of ${ }^{9} \mathrm{C}$ projectiles in each experiment was determined to be within $5 \%$ of each other from the elastic-scattering angular distributions measured with the $\mathrm{CsI}(\mathrm{Tl})$ crystals.

In addition to ${ }^{9} \mathrm{C}$, the secondary beams in both experiments also contained an $\approx 40 \%$ contribution from ${ }^{6} \mathrm{Li}$ nuclei and $\lesssim 1 \%$ contributions from ${ }^{8} \mathrm{~B}$ and ${ }^{7} \mathrm{Li}$ contaminants.

\section{Invariant-mass spectra}

The ${ }^{8} \mathrm{~B}$ excitation-energy spectra, determined for the detected $p+{ }^{7} \mathrm{Be}, 2 p+{ }^{6} \mathrm{Li}$ channels with the invariantmass technique, are shown in Figs. 2(a) and 2(b), respectively. The ordinate of these plots, $E_{n \gamma}^{*}$, is the excitation energy calculated assuming the ${ }^{7} \mathrm{Be}$ and ${ }^{6} \mathrm{Li}$ fragments were produced in their ground-states. However, in both these nuclei, there is one exited state that decays predominately by $\gamma$-ray emission. If single- or two-proton decays of ${ }^{8} \mathrm{~B}$ lead to the production of one these particlebound excited states, then the actual excitation of the ${ }^{8} \mathrm{~B}$ states is greater than $E_{n \gamma}^{*}$ by the energy of this $\gamma$ ray.

The $p+{ }^{7} \mathrm{Be}$ excitation-energy spectrum in Fig. 2(a) displays two strong peaks associated with the first $\left(J^{\pi} ; T=1^{+} ; 1\right)$ and second $\left(2^{+} ; 1\right)$ excited states of ${ }^{8} \mathrm{~B}$ at 0.770 and $2.320 \mathrm{MeV}$, respectively [22]. The level scheme of ${ }^{8} \mathrm{~B}$ and the decay paths of the excited states of interest in this work are presented in Fig. 3. Both of these peaks correspond to proton decays to the ground state of ${ }^{7} \mathrm{Be}$. The $1^{+} ; 1$ state also has a small proton decay branch $(\approx 1 \%)$ to the first-excited state of ${ }^{7} \mathrm{Be}[23]$. As the $\gamma$-ray energy is not included in the invariant-mass 

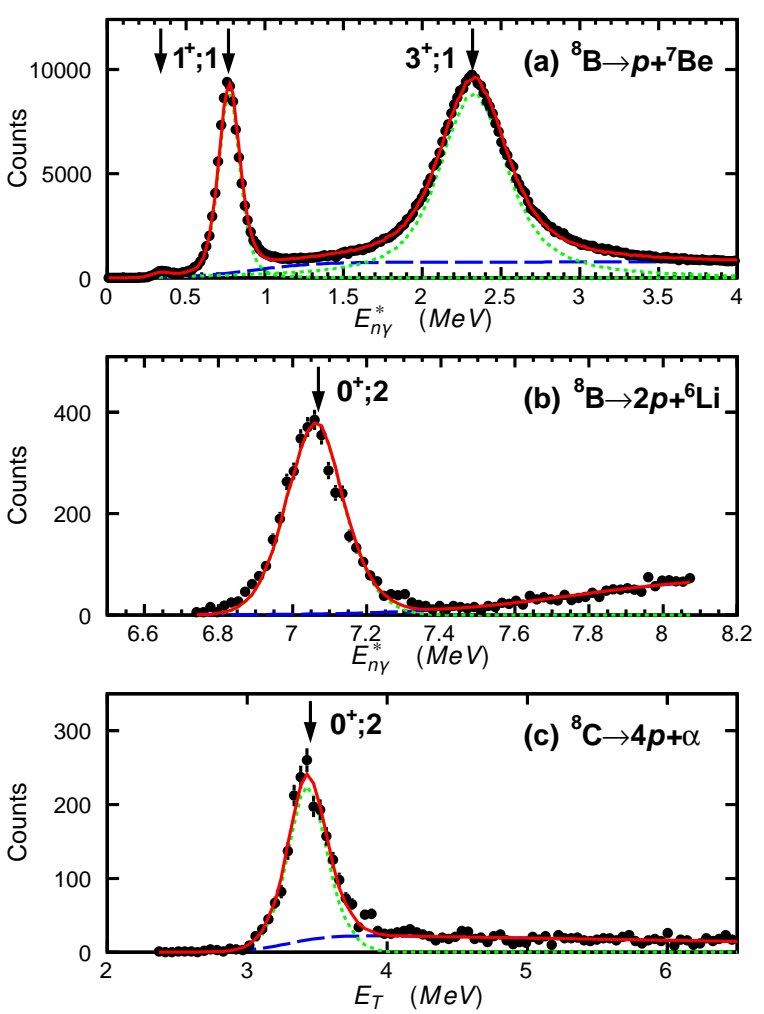

FIG. 2. Distribution of the excitation-energy $E_{n \gamma}^{*}$ and the total decay kinetic energy $E_{T}$ obtained with the invariant-mass method for the indicated exit-channels with the $E / A=66.8$ $\mathrm{MeV}{ }^{9} \mathrm{C}$ beam. The experimental distribution is shown by the data points, while the red curves give the fitted distribution which comprises contributions from the individual levels (green dotted curves) and a background (blue dashed curves). Energies of known states are indicated by the arrows.

measurement, this branch is visible as a very small peak just lower in energy to the main $1^{+} ; 1$ peak.

The $2 p+{ }^{6} \mathrm{Li}$ excitation spectrum in Fig. 2(b) displays a peak associated with the $0^{+} ; 2$ isobaric analog state (IAS) in ${ }^{8} \mathrm{~B}$ at $E^{*}=10.619 \mathrm{MeV}$ [21]. It has been shown that this state decays by prompt two-proton emission to the $T=1$ isobaric analog state in ${ }^{6} \mathrm{Li}$, which subsequently decays by the emission of a $3.562-\mathrm{MeV} \gamma$ ray. Thus this peak is located at $E_{n \gamma}^{*}=10.619-3.562=7.057 \mathrm{MeV}$.

The decay-energy spectrum for the $4 p+\alpha$ channel in Fig. 2(c) shows a clear peak associated with the $0^{+} ; 2$ ground state of ${ }^{8} \mathrm{C}$. It has been shown, from correlations between the momentum of the decay products determined in this data set, that this state undergoes a prompt two-proton decay to ${ }^{6} \mathrm{Be}^{g . s}$. which subsequently two-proton decays to $\alpha+p+p[16,20]$.

Nucleon knockout to the ${ }^{8} \mathrm{C} 0^{+} ; 2$ state and the ${ }^{8} \mathrm{~B} 0^{+} ; 2$ and $3^{+} ; 1$ states involves the removal of a $p_{3 / 2}$ nucleon. For the creation of the ${ }^{8} \mathrm{~B} 2^{+} ; 1$ ground state and the $1^{+} ; 1$ first-excited state, it is also possible that there are contributions from $p_{1 / 2}$-proton removal, but spectroscopic factors calculated with the shell model and the Variational Monte Carlo Model (Sec.IV) suggest the $p_{1 / 2}$ component

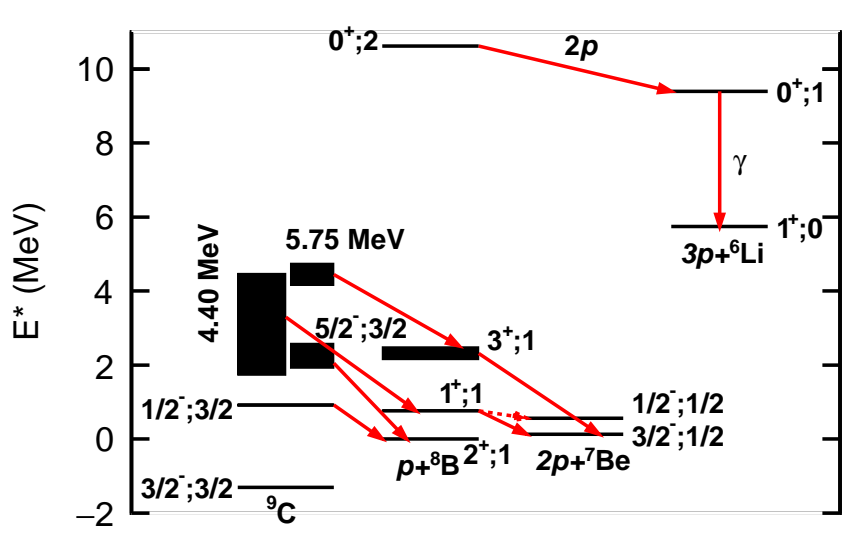

FIG. 3. Level scheme of ${ }^{8} \mathrm{~B}$ showing the states of interest in this work, their proton decay pathways, and the observed sequential proton feeding from the ${ }^{8} \mathrm{~B}$ levels.

is quite small. Thus, the expectation is that all these channels are predominately associated with $p_{3 / 2}$ nucleon removal.

\section{Feeding from proton-decays}

The ${ }^{8} \mathrm{~B}$ yields can include, in addition to the main knockout contribution, feeding from inelastically-excited ${ }^{9} \mathrm{C}$ projectiles which subsequently decay by proton emission. This pathway can also be studied with the invariant-mass technique and ${ }^{9} \mathrm{C}$ excited states which decay to, or through, ${ }^{8} \mathrm{~B}$ states were identified in Ref. [23] from this data set. The level diagram of Fig. 3 summaries these observed decay paths. The ground state of ${ }^{8} \mathrm{~B}$ is fed by the first $\left(1 / 2^{-} ; 3 / 2\right)$ and second $\left(5 / 2^{-} ; 3 / 2\right)$ excited states of ${ }^{9} \mathrm{C}$ at $E^{*}=2.218$ and $3.549 \mathrm{MeV}$, respectively, which were observed in the $p+{ }^{8} \mathrm{~B}$ invariant-mass spectra. In addition, the 4.40 and $5.75-\mathrm{MeV}$ states in ${ }^{9} \mathrm{C}$, which decay by sequential $2 p$ emission, feed the $1^{+} ; 1$ and $3^{+} ; 1$ states of ${ }^{8} \mathrm{~B}$, respectively. The contribution of this feeding to the observed yields is determined by simulating the production and decay of these ${ }^{9} \mathrm{C}$ states and adjusting their yields and angular distributions to fit the experimental data.

The simulated quantities depend on the fitted inelasticscattering angular distribution for each ${ }^{9} \mathrm{C}$ state. It was found that these depend on whether the target nucleus is excited or not. The target excitation energy $E_{\text {Target }}^{*}$ was determined assuming that the inelastic scattering follows two-body kinematics. Figure 4 shows the deduced distribution of $E_{\text {Target }}^{*}$ determined for all $p+{ }^{8} \mathrm{~B}$ events. It is similar in shape to that obtained for the $2 p+{ }^{7}$ Be events with a strong peak for $E_{\text {Target }}^{*}=0$ and a high-energy tail associated with higher target excitation energies or from events which do not follow two-body kinematics. The widths of the observed $E_{\text {Target }}^{*}$ peaks are consistent with the simulated resolution for this quantity. Similar-shaped 


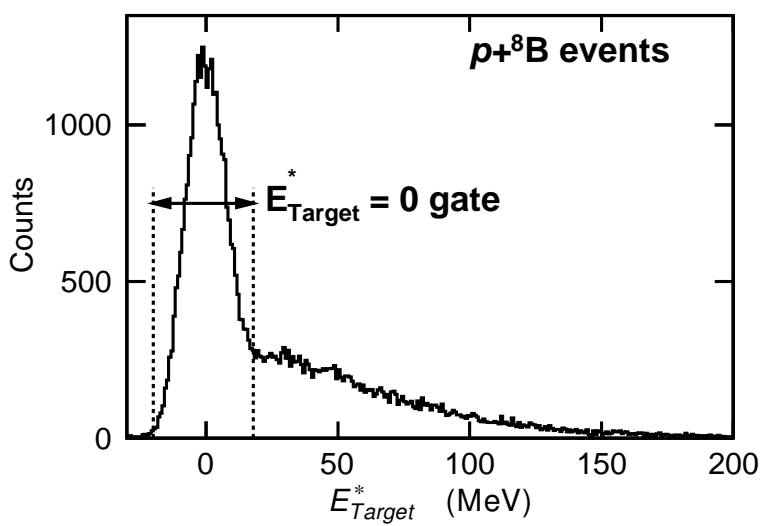

FIG. 4. Target-nucleus excitation-energy distribution obtained from detected ${ }^{9} \mathrm{C}$ projectile events that decay to the $p+{ }^{8} \mathrm{~B}$ exit channel. The excitation energy was deduced assuming two-body kinematics. The gate used to select $E_{\text {Target }}^{*}=0$ events is shown.

$E_{\text {Target }}^{*}$ distributions were observed for inelastic scattering of ${ }^{7} \mathrm{Be}(E / A=65.5 \mathrm{MeV})$ and ${ }^{6} \mathrm{Li}(E / A=36.6 \mathrm{MeV})$ projectiles on ${ }^{9} \mathrm{Be}$ targets [24] and for ${ }^{7} \mathrm{Li}$ projectiles on ${ }^{9} \mathrm{Be},{ }^{12} \mathrm{C}$, and ${ }^{27} \mathrm{Al}$ targets at $E / A=24 \mathrm{MeV}[25,26]$. The $E_{\text {Target }}^{*}=0$ component has a more forward focused angular distribution than that of the tail component.

The ${ }^{9} \mathrm{C}$ excitation-energy spectra for the $E_{\text {Target }}^{*}=0$ component (see gate in Fig. 4) are shown in Fig. 5. Panels (b) and (c) are from the $2 p+{ }^{7} \mathrm{Be}$ events which have been gated on the ${ }^{8} \mathrm{~B}_{1^{+;} ; 1}$ and ${ }^{8} \mathrm{~B}_{3^{+} ; 1}$ intermediate states, as in [23]. The solid red lines show fits to the these spectra used to determine the yield of each ${ }^{9} \mathrm{C}$ state. Apart from the contribution of ${ }^{9} \mathrm{C}$ peaks (dotted green curves), the fits also include a smooth background (dashed blue curves). This background can contain contributions from non-resonant breakup, which includes proton knockout events where both the knockedout proton and the residual decay fragment are detected. For instance, in knockout of a proton to the ground state of ${ }^{8} \mathrm{~B}$ at the somewhat-higher beam energy of $E / A=97.9$ $\mathrm{MeV}$, Bazin et al. extracted a proton angular distribution for the elastic knockout process and extrapolated this to small angles using continuum-discretized coupled -channels calculations [27]. With this distribution, roughly $30 \%$ of these protons would lie within the maximum angle of $12^{\circ}$ subtended by HiRA and thus can contribute to this background if the core is also detected. In addition, the background can include contributions from unresolved, wide resonances. The latter should be included in the sequential-feeding component, but we cannot differentiate these from the non-resonant component. The excitation spectra from the $E_{\text {Target }}^{*}>0$ events are very similar in shape, but the extracted yields of the ${ }^{9} \mathrm{C}$ states are smaller.

No evidence of any sequential feeding is found for the ${ }^{8} \mathrm{~B}_{0^{+} ; 2}$ and ${ }^{8} \mathrm{C}_{0^{+} ; 2}$ states. In principle, it is possible for feeding following neutron decay of appropriate states for which these experiment are insensitive. However, for

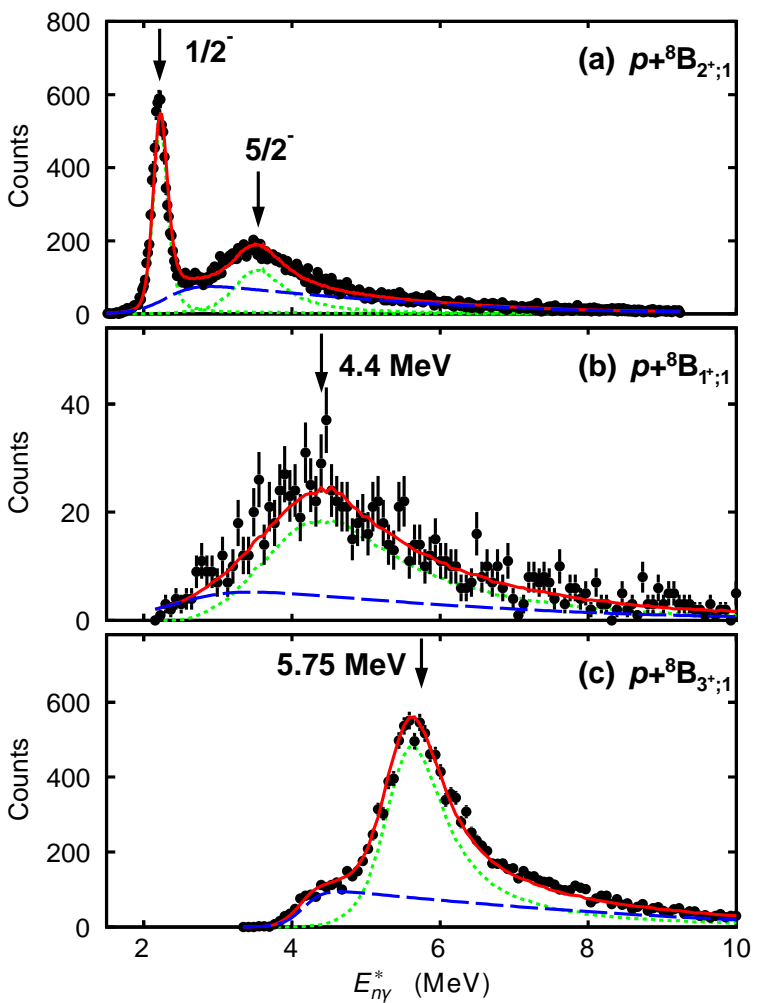

FIG. 5. Distributions of the excitation-energy $E_{n \gamma}^{*}$ obtained with the invariant-mass method for proton decay of inelastically-excited ${ }^{9} \mathrm{C}$ projectiles to the indicated states in ${ }^{8} \mathrm{~B}$, for $E_{\text {Target }}=0$. The solid red curves are fits to the data with contributions from individual levels (green dotted curves) and from backgrounds (blue-dashed curves).

such proton-rich systems we expect the contribution from neutron feeding to be minimal.

\section{Momentum and angular distributions}

The longitudinal-momentum distributions determined from the ${ }^{8} \mathrm{~B}$ and ${ }^{8} \mathrm{C}$ states are shown in Fig. 6. For the particle-unstable states, gates at the half-maximum points in the invariant-mass spectrum were used to reduce the relative contribution from background under the observed peaks. This is most important for the wide ${ }^{8} \mathrm{~B}_{3^{+} ; 1}$ state in Fig. 2(a). All $P_{\|}$distributions display a broad peak of similar width centered around the value corresponding to the beam velocity (vertical dotted lines). For the ${ }^{8} \mathrm{~B}$ ground state, in Fig. 6(a), a second narrow peak is also quite prominent at lower momentum. This peak is associated with elastic scattering of the small $(\lesssim 1 \%){ }^{8} \mathrm{~B}$ beam impurity. In subsequent analysis of these events, we have excluded the events in this peak and those of lower momentum. The final ${ }^{8} \mathrm{~B}_{2^{+} ; 1}$ yield is then increased by $27 \pm 5 \%$ to account for the missing tail events below our imposed lower cutoff. This correction is obtained from the relative fraction in the spectra for the other ${ }^{8} \mathrm{~B}$ states which are all similar 

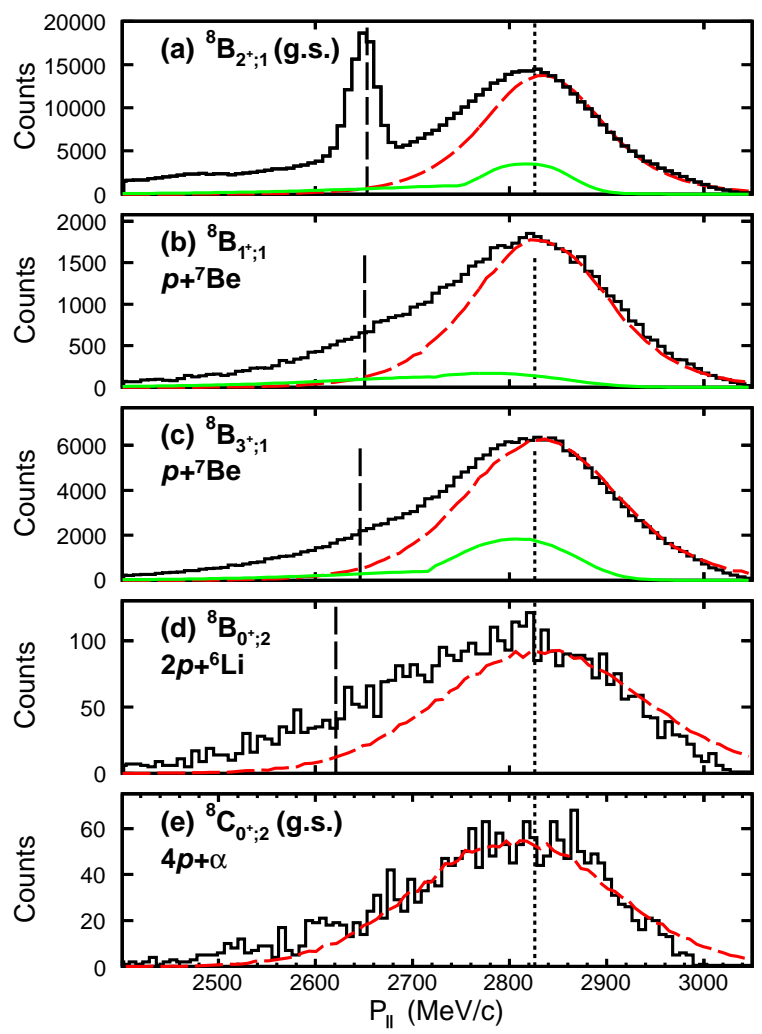

FIG. 6. Histograms showing the longitudinal-momentum distributions measured for the indicated residuals obtained with the $E / A=66.8-\mathrm{MeV}^{9} \mathrm{C}$ beam. Predictions from the eikonal calculations, normalized to the same peak height as the data, are shown by the red-dashed curves. The solid-green curves show the contributions from sequential proton feeding of inelastically excited ${ }^{9} \mathrm{C}$ projectiles. The vertical dotted lines show the momentum associated with the beam velocity at the center of the target, while the vertical dashed lines show the expected location of events from elastic and inelastically excited ${ }^{8} \mathrm{~B}$ projectiles (beam contaminant) for $E_{\text {Target }}=0$.

in magnitude to within the quoted uncertainty. In principle, inelastic scattering of the ${ }^{8} \mathrm{~B}$ beam impurity could also contribute to the tails of the excited ${ }^{8} \mathrm{~B}$ states, however this would produce peaks at the location indicted by the dashed vertical lines. As no such peaks are discerned, this contribution must be minimal.

The contributions to these $P_{\|}$distributions from sequential feeding as determined in the simulations of ${ }^{9} \mathrm{C}$ decay are shown by the solid green curves in Figs. 6(a) to $6(\mathrm{c})$. These distributions each have peak and tail components, reflecting the corresponding components in the $E_{\text {Target }}^{*}$ distributions (e.g. Fig. 4). The ${ }^{8} \mathrm{~B}_{3^{+} ; 1}$ and ${ }^{8} \mathrm{~B}_{2^{+} ; 1}$ states each have a $16 \%$ contribution from sequential feeding from proton decay. The contribution to the $1^{+} ; 1$ state is smaller, at $9 \%$. The feeding for the ${ }^{8} \mathrm{~B}_{3^{+} ; 1}$ state was amplified as two protons from the decay of the $5.75 \mathrm{MeV}$ state in ${ }^{9} \mathrm{C}$ are emitted with similar kinetic energies. If either one of these protons is not detected then the invariant mass of the other proton and the ${ }^{7} \mathrm{Be}$ residual contributes to the ${ }^{8} \mathrm{~B}_{3^{+} ; 1}$ peak in Fig. $2(\mathrm{a})$.

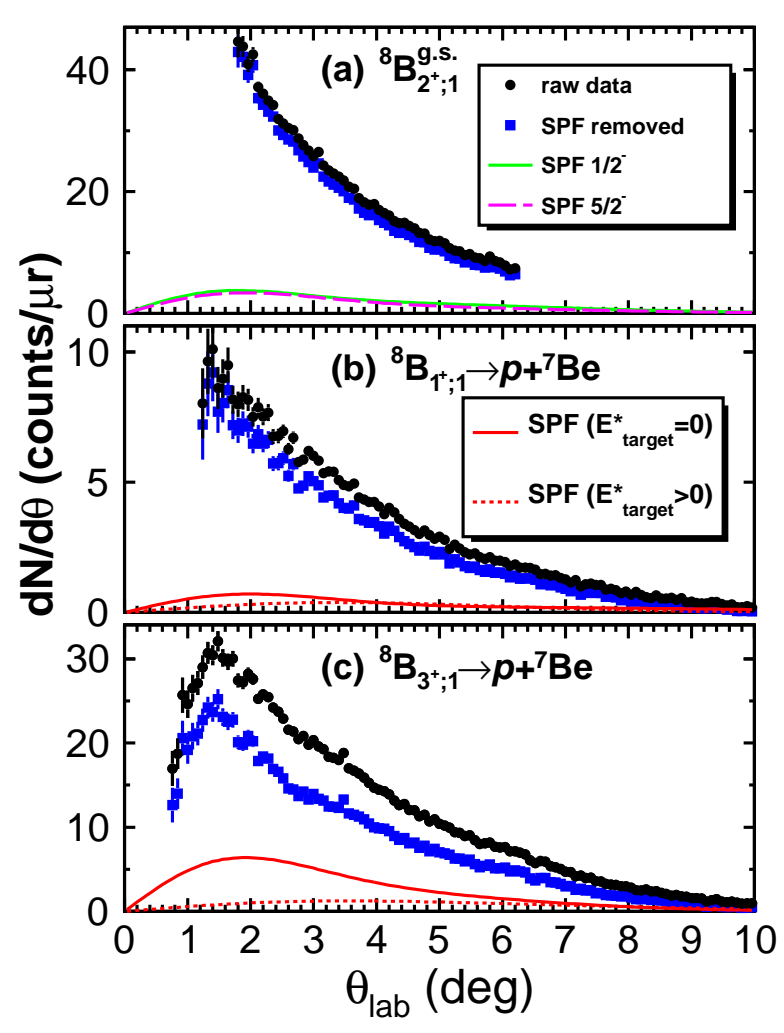

FIG. 7. Efficiency-corrected angular distributions in the laboratory frame obtained for the indicated residual states, and the $E / A=66.8-\mathrm{MeV}{ }^{9} \mathrm{C}$ beam, are shown as the black circular data points. Sequential proton feeding (SPF) contributions are indicated by the curves. In (a) these are subdivided into their contributions from the first $\left(1 / 2^{-}\right)$and second $\left(5 / 2^{-}\right)$exited states in ${ }^{9} \mathrm{C}$ for all $E_{\text {Target }}$. In (b) and (c) they are subdivided into their contributions from $E_{\text {target }}=0$ and $E_{\text {Target }}>0$. The blue square data points show the distributions after the subtraction of the SPF contributions.

Examples of the efficiency-corrected angular distributions $d N / d \theta$ of the knock-out products are shown in Fig. 7 , as circular data points. The efficiency is obtained from the Monte Carlo simulations assuming isotropic angular distributions as input.

The ${ }^{8} \mathrm{~B}_{2^{+} ; 1}$ ground-state distribution, in Fig. 7(a), was measured over a limited angular range as only the two inner-most telescopes had $\mathrm{Si}$ amplifier gains suitable to identify ${ }^{8} \mathrm{~B}$ fragments. The ${ }^{8} \mathrm{~B}_{3^{+} ; 1}$ result, in Fig. $7(\mathrm{c})$, covers a much larger angular range extending down to $1^{\circ}$ where the large kinetic-energy release in the proton decay can allow both decay products to recoil to larger angles and be detected.

The curves in Fig. 7 show the identified feeding contributions from proton decay of ${ }^{9} \mathrm{C}$ states. In Fig. $7(\mathrm{a})$, the contributions from the $1 / 2^{-}$and $5 / 2^{-}$states are indicted by the solid green and dashed blues curves. They are both similar in magnitude and angular dependence. In Figs. 7(b) and 7(c), the feeding contribution is shown for $E_{\text {Target }}^{*}=0$ (solid red) and $E_{\text {Target }}^{*}>0$ (dotted red). The $E_{\text {Target }}^{*}=0$ component is more intense and more forwardfocused in both cases. In all three panels of Fig. 7, the 
blue square data points show residual yields after these proton-feeding contributions are subtracted.

These angular distributions are an important input to the Monte Carlo simulations used to calculate the detection efficiencies for each of the observed channels. Alternatively, the total yield of these channels is the integral of the angular distributions corrected for the fraction of the yield outside the gate on the invariant-mass peaks. Figure 8 shows fits to all of the extracted efficiency-corrected, feeding-subtracted angular distributions. The largest uncertainty in the simulated efficiencies and the final yields is due to the extrapolation of these distributions to angular regions with no experimental data and thus some model is needed to make these extrapolations. We start by comparing the data and the simulations results (dotted green curves) using the transverse-momentum distributions predicted with our eikonal-model calculations (Sec. IV). These distributions include the effects of the angular resolution from the beam-spot size on the target and beam divergence, but this makes only a minor difference. For the cases where the experimental angular distributions are extracted down to small angles, such as the $J=3^{+} ; 1$ state of ${ }^{8} \mathrm{~B}$ and the ground state of ${ }^{8} \mathrm{C}$, in Figs. 8(c) and 8(e), respectively, the eikonal model predicts the location and width of the peaks in the experimental distributions quite well. However, these calculations do not predict enough relative yield in the largerangle tails. To compensate for this, we have added a second component into the simulations assuming transversemomentum distributions of the form

$$
\frac{d^{2} \sigma}{d P_{\perp}} \propto P_{\perp}^{n} \exp \left(-P_{\perp}^{2} / T\right)
$$

with fit parameters $n$ and $T$ plus the integrated yield of this component. The $n$ and $T$ parameters were constrained by fitting the $3^{+} ; 1^{8} \mathrm{~B}$ state, in Fig. $8(\mathrm{c})$, which has the most complete angular coverage with the best statistics. These parameters were kept fixed for the other states where only the relative contributions of the "tail" and the eikonal-model predictions were varied to best reproduce the data. The fits are consistent to the extent that the "tail" components account for $4-13 \%$ of the yield in all of the fits.

The final detection efficiencies, $\epsilon$, are listed in Table I. No absolute measurement of the number of projectiles incident on the target was made in these experiments. To normalize the cross sections, we note that the ${ }^{8} \mathrm{~B}_{2^{+} ; 1}^{\text {g.s. }}$ knockout yield from ${ }^{9} \mathrm{C}$ with a ${ }^{9} \mathrm{Be}$ target was measured at a somewhat higher beam energy of $E / A=97.9 \mathrm{MeV}$ [27]. As the knockout cross section has a modest energy dependence [28-30], we have used this value to fix the total cross section from both the true knockout reactions and the sequential feeding for the ${ }^{8} \mathrm{~B}_{2^{+;} ; 1}^{\text {g.s. }}$ state. The experimental cross section of $56(3) \mathrm{mb}$ at $E / A=97.9 \mathrm{MeV}$ was increased to $58 \mathrm{mb}$ to account for the relative increase in the calculated cross section predicted by the eikonal model at the lower beam energy. To highlight the relative differences between the channels, for the normalization of the cross sections we have not propagated our $15 \%$ uncer-

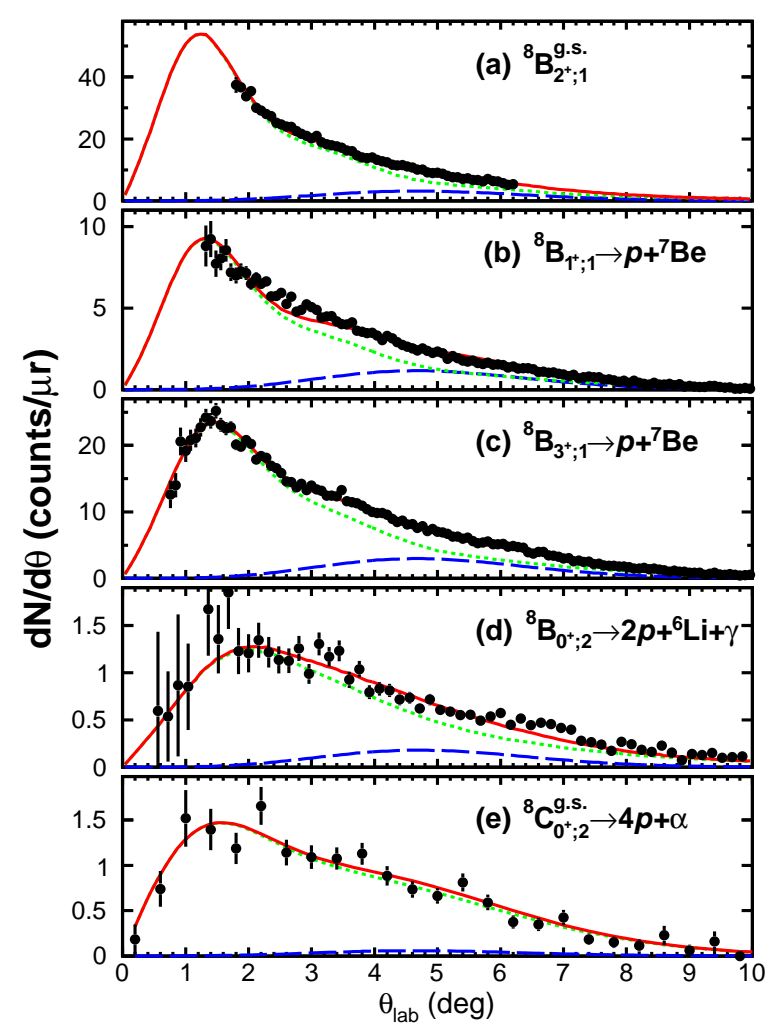

FIG. 8. Data points show the angular distributions of the indicated residuals in the laboratory frame with the sequential proton feeding subtracted. The solid red curves show fits to the data with a contribution that follows the angular dependence predicted by the eikonal model (green dotted curves) and a "tail" component (blue-dashed curves).

tainty in the determination of the ${ }^{8} \mathrm{~B}^{\text {g.s. }}$ yield or the $6 \%$ uncertainty in the ${ }^{8} \mathrm{~B}^{\text {g.s. }}$ cross section measured by Bazin et al. to the other channels. To obtain absolute uncertainties, an extra $21 \%$ systematic uncertainty must be added to the listed uncertainties for these channels. The knockout cross sections, $\sigma_{\text {exp }}$, for all channels with the sequential feeding subtracted are also listed in Table I.

\section{B. KNOCKOUT FROM ${ }^{13} \mathrm{O}$}

The HiRA setup, with an identical configuration, was also used in an experiment with a ${ }^{13} \mathrm{O}$ secondary beam of energy of $E / A=65.4 \mathrm{MeV}$ incident on the same 1-mmthick ${ }^{9}$ Be target. Details of the experiment can be found in Ref. [13]. New dual-gain amplifiers for the silicon detectors were used to allow all particle-types of interest to be identified in all telescopes. The beam purity was $95 \%$. In this study, in addition to particle-unstable states, we also include the proton knockout to the particle-stable ${ }^{12} \mathrm{~N}$ ground state for which, once again, we expect no sequential $\gamma$ decay feeding. 
TABLE I. Results for the residual produced in single-nucleon knockout reactions from the $E / A=66.8-\mathrm{MeV}^{9} \mathrm{C}$ beam. These include the spin/isospin $J^{\pi} ; \mathrm{T}$, excitation energy $E^{*}$ and particle decay width $\Gamma$ of the residual, the separation energy $E_{\text {sep }}$ of the removed nucleon, the efficiency $\epsilon$ for detection of the decay products, the sequential proton feeding fraction SPF, the experimental knockout cross section $\sigma_{\text {exp }}$ with the feeding removed, the single-particle cross section $\sigma_{\text {s.p. }}$. from the eikonal calculation (using the Hartree-Fock (HF) rms radius to constrain the overlap) and from the overlap function predicted with the VMC model, the spectroscopic factors $C^{2} S$ obtained with the Cohen-Kurath (CK) interactions and their equivalent in the VMC model, the final theoretical cross sections $\sigma_{C K, V M C}$ from both models, and the ratios of experimental to theoretical cross sections $\sigma_{\text {exp }} / \sigma_{C K, V M C}$.

\begin{tabular}{cccccc}
\hline \hline nucleon & $p$ & $p$ & $p$ & $p$ & $n$ \\
residual & ${ }^{8} \mathrm{~B}$ & ${ }^{8} \mathrm{~B}$ & ${ }^{8} \mathrm{~B}$ & ${ }^{8} \mathrm{~B}$ & ${ }^{8} \mathrm{C}$ \\
decay channel & ${ }^{8} \mathrm{~B}$ & $p+{ }^{7} \mathrm{Be}$ & $p+{ }^{7} \mathrm{Be}$ & $2 p+{ }^{6} \mathrm{Li}+\gamma$ & $4 p+\alpha$ \\
$J^{\pi} ; T$ & $2^{+} ; 1$ & $1^{+} ; 1$ & $3{ }^{+} ; 1$ & $0^{+} ; 2$ & $0^{+} ; 2$ \\
$E^{*}(\mathrm{MeV})$ & 0 & 0.770 & 2.320 & 10.619 & 0 \\
$\Gamma(\mathrm{keV})$ & 0 & $35.6(6)$ & $350(3)$ & $<60$ & $130(10)$ \\
$E_{\text {sep }}(\mathrm{MeV})$ & 1.296 & 2.066 & 3.616 & 11.915 & 14.225 \\
$\epsilon(\%)$ & $10.7(12)$ & $8.5(7)$ & $8.9(5)$ & $3.2(4)$ & $0.61(11)$ \\
$\mathrm{SPF}(\%)$ & $16.6(8)$ & $8.8(4)$ & $16.4(8)$ & & \\
$\sigma_{\text {exp }}(\mathrm{mb})$ & $48.6(73)$ & $12.1(11)$ & $37.7(24)$ & $1.60(22)$ & $3.93(88)$ \\
$C^{2} S_{C K}$ & 0.909 & 0.363 & 1.48 & 0.243 & 0.974 \\
$\sigma_{s . p .}^{H . F}(\mathrm{mb})$ & 63.7 & 55.9 & 46.3 & 28.8 & 32.1 \\
$\sigma_{C K}(\mathrm{mb})$ & 58.0 & 20.3 & 68.4 & 7.01 & 31.3 \\
$\sigma_{\text {exp }} / \sigma_{C K}$ & $0.84(13)$ & $0.60(5)$ & $0.55(3)$ & $0.23(3)$ & $0.12(3)$ \\
\hline$C^{2} S_{V M C}$ & 0.995 & 0.469 & 1.266 & 0.16 & 0.632 \\
$\sigma_{s . p .}^{V M C}(\mathrm{mb})$ & 57.0 & 52.2 & 49.4 & 38.0 & 34.9 \\
$\sigma_{V M C}$ & 56.7 & 24.4 & 62.5 & 6.08 & 22.06 \\
$\sigma_{\text {exp }} / \sigma_{V M C}$ & $0.86(13)$ & $0.49(4)$ & $0.60(4)$ & $0.26(4)$ & $0.18(4)$ \\
\hline \hline
\end{tabular}

\section{Invariant-mass spectra}

The states produced in ${ }^{12} \mathrm{~N}$ and ${ }^{12} \mathrm{O}$ by single nucleon knockout are shown in the level diagram of Fig. 9 along with their decay pathways. Single-proton knockout reactions producing the ground state $\left(1^{+} ; 1\right)$ and the first excited state $\left(2^{+} ; 1, E^{*}=0.961 \mathrm{MeV}\right)$ of ${ }^{12} \mathrm{~N}$ are expected to be dominated by the knockout of a $p_{1 / 2}$ proton, based on shell-model predictions. The $2^{+} ; 1$ first-excited state decays by single-proton emission. The excitation-energy spectrum for $p+{ }^{11} \mathrm{C}$ events, shown in Fig. 10(a), displays a strong peak associated with this excited state. A small high-energy shoulder, associated with the second excited state $\left(2^{-} ; 1\right)$ is also present. The solid curve shows the fit used to determine the number of detected events from the $2^{+} ; 1$ state. We have not attempted to extract a cross section for the $2^{-} ; 1$ state as it has a small peak-to-background ratio making extraction of its angular distributions uncertain.

The neutron knockout reaction also populated the ${ }^{12} \mathrm{O}^{g . s}$ channel, the isobaric analog of which lies at $12.242 \mathrm{MeV}$ in ${ }^{12} \mathrm{~B}$ and is the second known $0^{+}$state

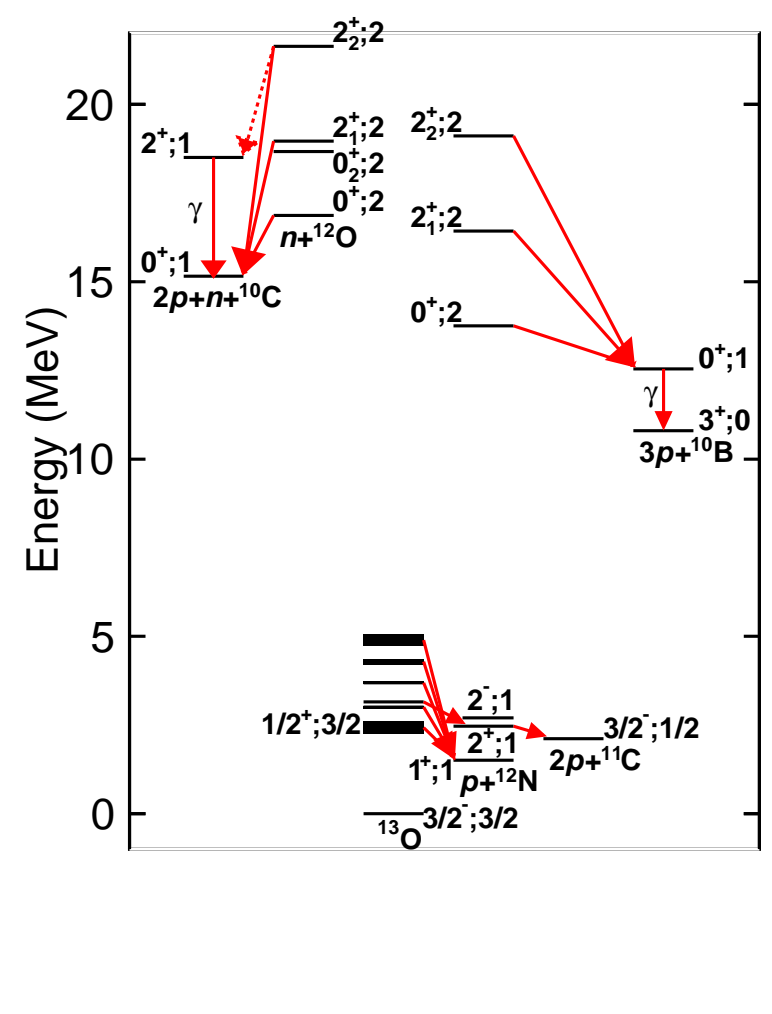

FIG. 9. Level diagram showing states produced via one nucleon knockout from the ${ }^{13} \mathrm{O}$ beam. The arrows show their one and two-proton decay paths observed in the invariantmass spectra. Inelastically-excited states in ${ }^{13} \mathrm{O}$ which feed the low-energy states in ${ }^{12} \mathrm{~N}$ by proton decay are also included.

in ${ }^{12} \mathrm{~N}$. Production of both of these states involves the removal of a $p_{3 / 2}$ nucleon from ${ }^{13} \mathrm{O}$ and both states decay by two-proton emission [13]. They are observed with minimal background in the invariant-mass spectra in Figs. 10(b) and 10(c) where fits used to extract the detected yields are also shown. The IAS in ${ }^{12} \mathrm{~N} 2 p$ decays to the IAS in ${ }^{10} \mathrm{~B}$, which subsequently emits a $1.740-$ $\mathrm{MeV} \gamma$ ray $[13,31]$. So, it is associated with the peak at $\mathrm{E}_{n \gamma}^{*}=12.242-1.740=10.502 \mathrm{MeV}$ in Fig. 10(b). Also present in these invariant-mass spectra are the peaks associated with $J^{\pi}=2^{+}, T=2$ analogs in ${ }^{12} \mathrm{O}$ and ${ }^{12} \mathrm{~N}$. There are two such states in each spectrum which are labeled by $2_{1}^{+}$and $2_{2}^{+}$. The subscripts here refer to the first and second $J^{\pi}=2^{+} ; T=2$ state observed in each isotope. We note that there are significant backgrounds under all of the $2^{+} ; 2$ state peaks so this adds some uncertainty in determining their angular distributions. To reduce this uncertainty, tighter gates $(\approx \pm 300 \mathrm{keV})$ were placed around each peak maximum when extracting the angular distributions. We note however that gates placed on the tails of the peaks produced similar-shaped angular distributions so the error associated with background within these gates is expected to be small. 

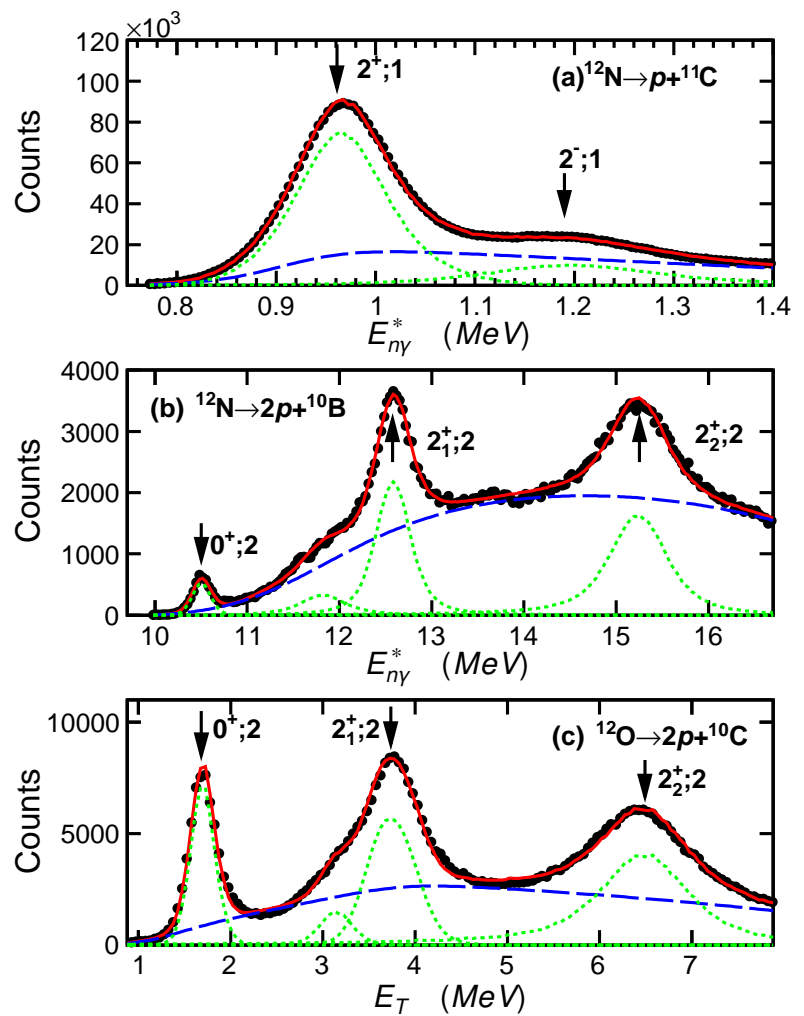

FIG. 10. Distributions of the excitation-energy, $E_{n \gamma}^{*}$, and total decay kinetic energy, $E_{T}$, for residuals produced following single-nucleon knockout reactions from the $E / A=65.4-\mathrm{MeV}$ ${ }^{13} \mathrm{O}$ beam. The curves are as described in the caption of Fig. 2.

\section{Feeding from proton decay}

Sequential proton feeding to the ground state of ${ }^{12} \mathrm{~N}$ was also observed from inelastically excited states of ${ }^{13} \mathrm{O}$. The excitation-energy spectrum obtained from $p+{ }^{12} \mathrm{~N}$ events with $E_{\text {Target }}^{*}=0$ is shown in Fig. 11 where it is fit with five peaks plus a smooth background. The most prominent peak, at $\mathrm{E}^{*}=2.956(10) \mathrm{MeV}$, has been observed in a previous invariant-mass study [32] and the lowest-energy peak is the $1 / 2^{+}$first-excited state observed in $p+{ }^{12} \mathrm{~N}$ scattering [33]. For some of these states, the angular distributions of the decay fragments in the parent's reference frame for the $E_{\text {Target }}^{*}=0$ component were found to be strongly anisotropic. This is indicative of significant ${ }^{13} \mathrm{O}^{*}$ spin alignment similar to that found in Refs. [24-26, 34]. More details will be given in a future paper on the structure of ${ }^{13} \mathrm{O}$. Monte Carlo simulations were fitted to these data and then used to determine the sequential feeding as in the analysis of ${ }^{9} \mathrm{C}$ inelastic scattering in Sec. II A 2. This feeding was found to contribute $21(1) \%$ of the detected ${ }^{12} \mathrm{~N}_{1^{+} ; 1}^{g . s}$ fragments. The ${ }^{12} \mathrm{~N}_{2^{+} ; 1}$ state yield was found to have an $\approx 1 \%$ feeding from the $E^{*}=3.025-\mathrm{MeV}$ level observed in the $2 p+{ }^{11} \mathrm{C}$ invariant-mass spectrum [32] which sequentially $2 p$ decays through this state. No feeding was identified for the other ${ }^{12} \mathrm{~N}$ and ${ }^{12} \mathrm{O}$ states.

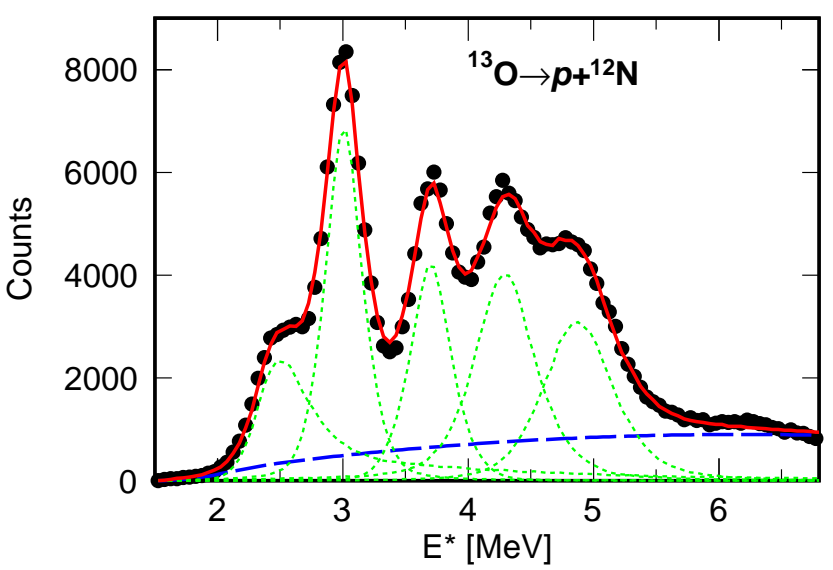

FIG. 11. Excitation-energy distribution showing fitted states in ${ }^{13} \mathrm{O}$ that proton decay to the ground state of ${ }^{12} \mathrm{~N}$. The curves are as described in the caption of Fig. 2.

\section{Momentum and angular distributions}

The longitudinal-momentum distributions determined for six of the considered states are shown in Fig. 12. A low-momentum threshold has been applied to the ${ }^{12} \mathrm{~N}_{1+; 1}^{\text {g.s. }}$ spectrum as the ${ }^{12} \mathrm{~N} Z$-line is crossed by an artifact in the $E-\Delta E$ plot - associated with elastic scattering in the inner detectors. A correction of $9 \%$, based on an extrapolation using the ${ }^{12} \mathrm{~N}_{2^{+} ; 1}$ spectral shape [Fig. 12(b)], was applied to account for the missing tail. The dotted magenta curve shows this extrapolation in Fig. 12(a).

The efficiency-corrected $d N / d \theta$ distributions with sequential proton feeding subtracted are displayed in Fig. 13. We have again made the same model-dependent extrapolations to zero angle as in Sec. II A 3 to account for the missing acceptance of the detector. The yield at smaller angle is assumed to be described by the eikonal model prediction with a "tail" component added to reproduce the data. We investigated different values of the parameter $n$ in Eq. (2) in the fits and this range of values is captured by the quoted errors on the efficiencies.

The secondary beam in this experiment was further purified using the radio frequency fragment separator [35] where a scintillation foil was used to detect separate beam particles. Corrections due to losses of beam particles in transport to the target were determined from periodically placing a $\mathrm{CsI}(\mathrm{Tl})$ scintillation counter in the target position during the experiments. Corrections were also made for the acquisition dead-time using a random pulse generator. The final number of beam particles is determined to $10 \%$ and the target thickness is known to $2 \%$. With these values, the exclusive cross sections of the knockout products are given in Table II. 
TABLE II. Results for single-nucleon knockout reactions from the $E / A=65.4-\mathrm{MeV}{ }^{13} \mathrm{O}$ and the $E / A=58.2-\mathrm{MeV}{ }^{17} \mathrm{Ne}$ beams. Quantities are the same as were detailed in Table I.

\begin{tabular}{|c|c|c|c|c|c|c|c|c|c|c|}
\hline beam & ${ }^{13} \mathrm{O}$ & ${ }^{13} \mathrm{O}$ & ${ }^{13} \mathrm{O}$ & ${ }^{13} \mathrm{O}$ & ${ }^{13} \mathrm{O}$ & ${ }^{13} \mathrm{O}$ & ${ }^{13} \mathrm{O}$ & ${ }^{13} \mathrm{O}$ & ${ }^{17} \mathrm{Ne}$ & ${ }^{17} \mathrm{Ne}$ \\
\hline nucleon & $p$ & $p$ & $p$ & $p$ & $p$ & $n$ & $n$ & $n$ & $n$ & \\
\hline residal & ${ }^{12} \mathrm{~N}$ & ${ }^{12} \mathrm{~N}$ & ${ }^{12} \mathrm{~N}$ & ${ }^{12} \mathrm{~N}$ & ${ }^{12} \mathrm{~N}$ & ${ }^{12} \mathrm{O}$ & ${ }^{12} \mathrm{O}$ & ${ }^{12} \mathrm{O}$ & ${ }^{16} \mathrm{Ne}$ & ${ }^{16} \mathrm{Ne}$ \\
\hline decay channel & ${ }^{12} \mathrm{~N}$ & $p+{ }^{11} \mathrm{C}$ & $2 p+{ }^{10} \mathrm{~B}+\gamma$ & $2 p+{ }^{10} \mathrm{~B}+\gamma$ & $2 p+{ }^{10} \mathrm{~B}+\gamma$ & $2 p+{ }^{10} \mathrm{C}$ & $2 p+{ }^{10} \mathrm{C}$ & $2 p+{ }^{10} \mathrm{C}$ & $2 p+{ }^{14} \mathrm{O}$ & $2 p+{ }^{14} \mathrm{O}$ \\
\hline$J^{\pi} ; \mathrm{T}$ & $1^{+} ; 1$ & $2^{+} ; 1$ & $0^{+} ; 2$ & $2_{1}^{+} ; 2$ & $2_{2}^{+} ; 2$ & $0^{+} ; 2$ & $2_{1}^{+} ; 2$ & $2_{2}^{+} ; 2$ & $0^{+} ; 2$ & $2^{+} ; 2$ \\
\hline$E^{*}(\mathrm{MeV})$ & 0 & 0.961 & 12.242 & 14.314 & 16.992 & 0 & 2.099(18) & $4.775(17)$ & 0 & $1.69(2)$ \\
\hline$\Gamma(\mathrm{keV})$ & 0 & $<20$ & $<100$ & $193(26)$ & $420(120)$ & $51(19)$ & $155(15)$ & $754(25)$ & $<80$ & $150(50)$ \\
\hline$E_{\text {sep }}(\mathrm{MeV})$ & 1.512 & 2.473 & 13.754 & 15.826 & 18.504 & 16.87 & 18.97 & 21.65 & 15.558 & 17.25 \\
\hline$\epsilon(\%)$ & $21.5(3.2)$ & $6.8(11)$ & $3.26(19)$ & $4.38(36)$ & $3.62(30)$ & $3.98(64)$ & $4.41(36)$ & $3.25(27)$ & $2.29(18)$ & $2.67(21)$ \\
\hline $\mathrm{SPF}(\%)$ & $21(1)$ & $1.2(5)$ & & & & & & & & \\
\hline$\sigma_{\text {exp }}(\mathrm{mb})$ & 34.(12) & $30.2(89)$ & $0.138(29)$ & $0.93(22)$ & $1.36(29)$ & $1.35(44)$ & $2.25(66)$ & $4.26(90)$ & $3.96(44)$ & $1.7(4)$ \\
\hline$C^{2} S$ & 0.666 & 1.04 & 0.139 & 0.465 & 0.163 & 0.556 & 1.86 & 0.654 & 0.875 & .0792 \\
\hline$\sigma_{s . p .}(\mathrm{mb})$ & 50.5 & 43.6 & 22.4 & 20.2 & 18.8 & 20.5 & 19.3 & 18.1 & 18.1 & 17.0 \\
\hline$\sigma_{t h}(\mathrm{mb})$ & 33.6 & 45.3 & 3.12 & 9.40 & 3.07 & 11.4 & 35.9 & 11.8 & 15.8 & 1.35 \\
\hline$\sigma_{e x p} / \sigma_{t h}$ & $1.0(4)$ & $0.67(2)$ & $0.044(9)$ & $0.099(22)$ & $0.44(9)$ & $0.12(4)$ & $0.063(11)$ & $0.36(3)$ & $0.25(6)$ & $1.28(14)$ \\
\hline
\end{tabular}

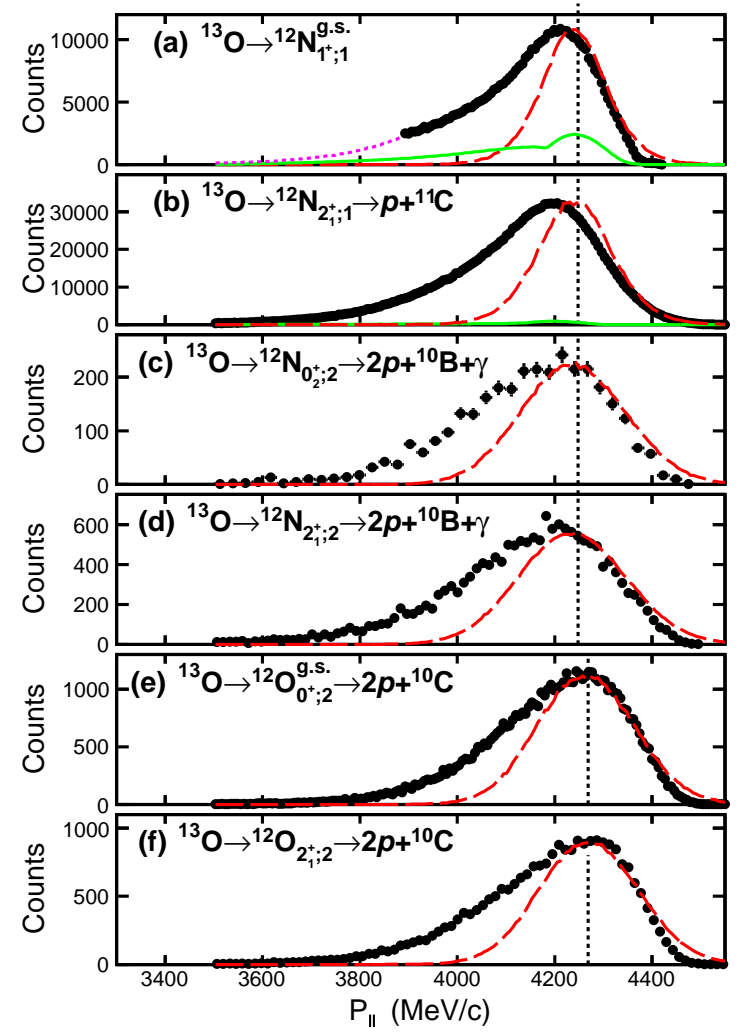

FIG. 12. Longitudinal-momentum distributions, as in Fig. 6, but for reactions of the $E / A=65.4-\mathrm{MeV}^{13} \mathrm{O}$ beam. The magenta dotted curve in (a) shows the extrapolation used to account for the missing yield below the low-momentum threshold for the identification of ${ }^{12} \mathrm{~N}$ fragments.

\section{KNOCKOUT FROM ${ }^{17} \mathrm{Ne}$}

Data obtained from earlier invariant-mass studies with an $E / A=58.2-\mathrm{MeV}{ }^{17} \mathrm{Ne}$ beam $[12,14,34]$ were also

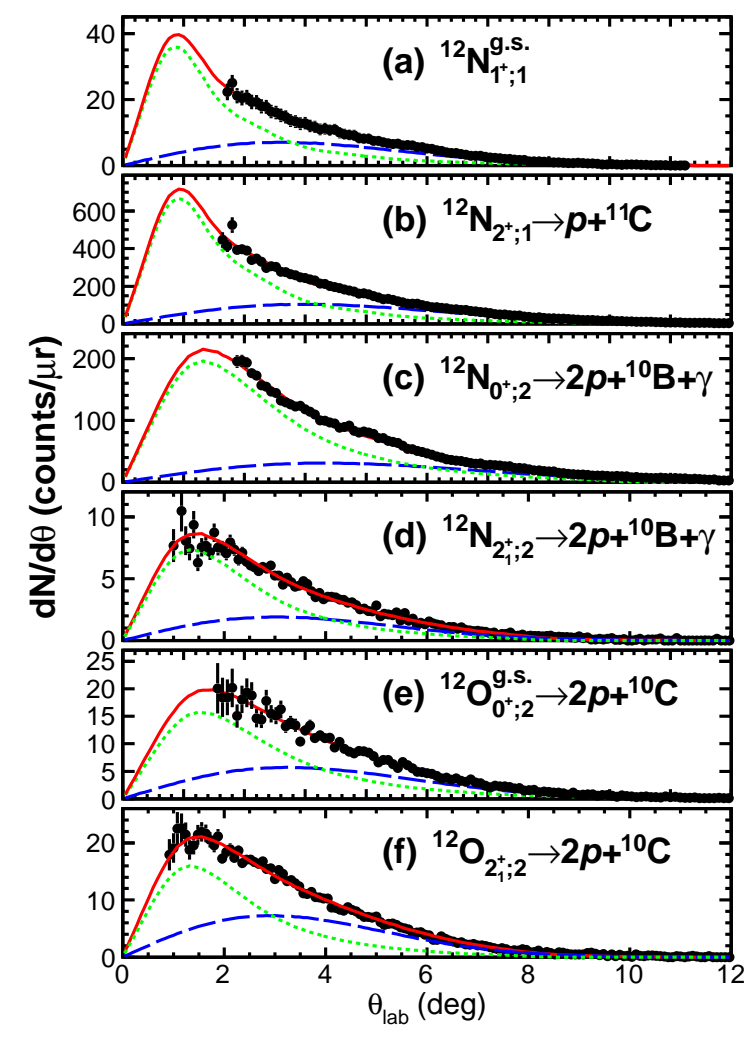

FIG. 13. Efficiency-corrected angular distributions in the laboratory frame with the sequential proton feeding removed, as in Fig. 8, but for the $E / A=65.4-\mathrm{MeV}^{13} \mathrm{O}$ beam.

employed. Only results for neutron knockout to ${ }^{16} \mathrm{Ne}$ will be presented as the proton knockout channels are difficult to separate. The decay-energy spectrum from $2 p+{ }^{14} \mathrm{O}$ events, in Fig. 14, shows the presence of the $0^{+} ; 2$ ground state of ${ }^{16} \mathrm{Ne}$ and its $2^{+} ; 2$ first excited state. The efficiency-corrected ground-state angular distribu- 


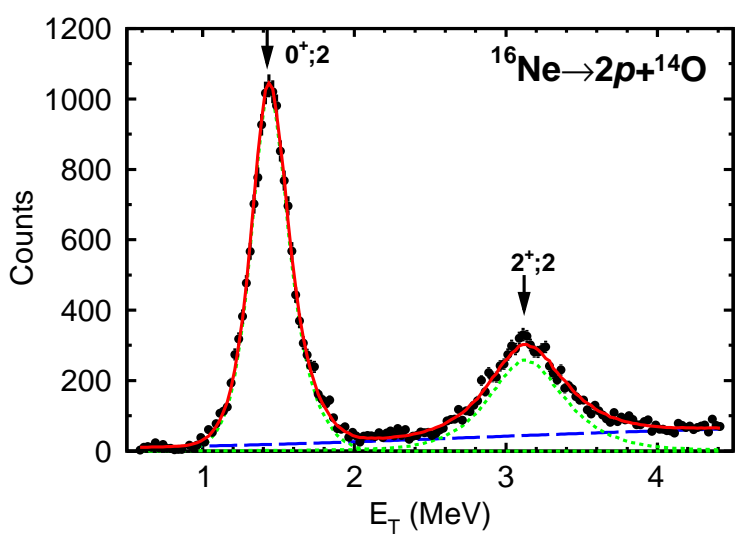

FIG. 14. Distribution of the decay energy, $E_{T}$, for the $2 p+{ }^{14} \mathrm{O}$ exit channel populated from the $E / A=58.2-\mathrm{MeV}^{17} \mathrm{Ne}$ beam.

tion and longitudinal-momentum distribution are shown in Figs. 15(a) and 15(b), respectively. They are qualitatively similar to the other neutron knockout distributions. With the larger mass of the projectile, the angular distribution does not extend out as far in angle as found for the lighter projectiles. The solid red curve in Fig. 15(a) shows the fit to this distribution with the two components. The relative weights are roughly similar to that found for the ${ }^{13} \mathrm{O}$ knockout channels.

The cross sections were normalized using the number of beam particles detected with a thin scintillation foil at the focal point of the A1900 spectrometer. Again corrections for the data-acquisition dead time, the beam purity, and its transport efficiency to the target position were made. The latter enabled by temporarily placing a detector in the target position. The simulated detector efficiency and deduced cross section are also listed in Table II.

\section{III. $28 \mathrm{MEV} / \mathrm{A}$ DATA}

Knockout cross sections are not expected to have a strong bombarding energy dependence [28-30]. We can check this for the particle-unstable residuals by using data for the ${ }^{13} \mathrm{O} \rightarrow{ }^{12} \mathrm{O}$ reaction which was also measured at $E / A=28.2 \mathrm{MeV}$, at the Texas A\&M University $\mathrm{Cy}$ clotron Facility [31]. This beam energy is too low to apply the eikonal-model and the sudden approximation, but a significantly larger cross section from these data would cast doubt upon the value obtained at the higher beam energy.

The $28.2 \mathrm{MeV} / A^{13} \mathrm{O}$ secondary beam $\left(2000-4000 \mathrm{~s}^{-1}\right)$ impinged on a $45.6 \mathrm{mg} / \mathrm{cm}^{-2}{ }^{9} \mathrm{Be}$ target and the ${ }^{12} \mathrm{O}$ decay products and the unreacted ${ }^{13} \mathrm{O}$ beam particles were detected in a single large-area $\mathrm{Si}-\mathrm{CsI}(\mathrm{Tl}) E-\Delta E$ telescope centered on the beam axis. See Ref. [31] for more details. The $E$ detector consisted of 32 closepacked $\mathrm{CsI}(\mathrm{Tl})$ crystals to allow for multi-hit capability. This telescope covered most of the angular spread of the decay fragments and so there is less sensitiv-
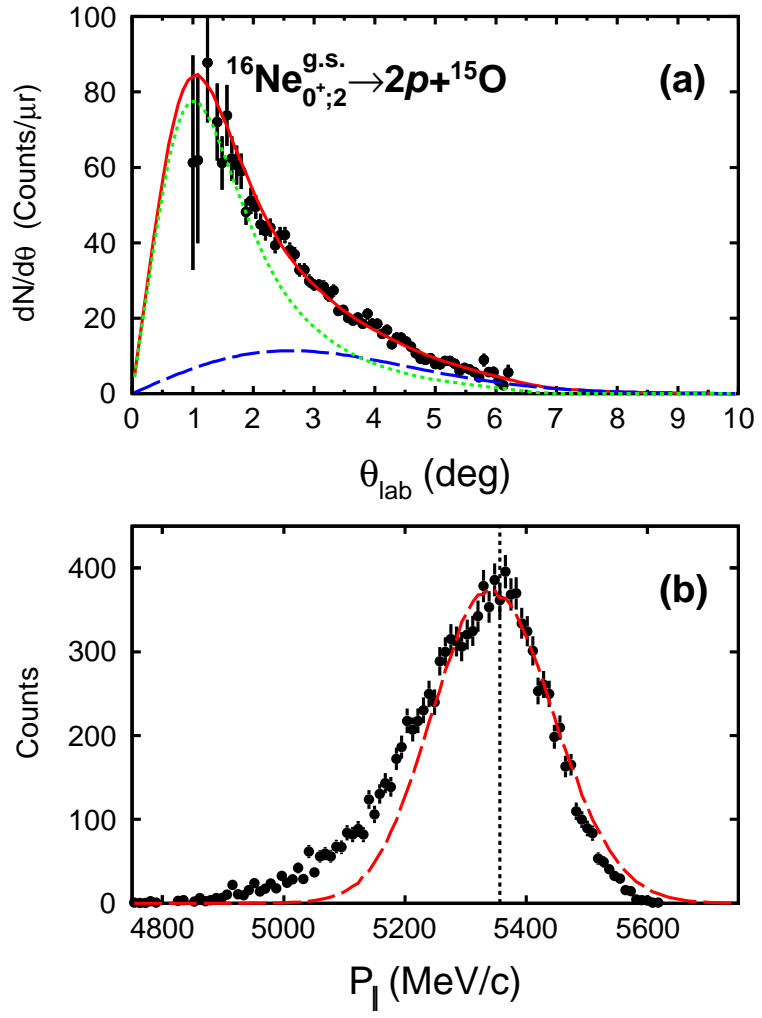

FIG. 15. (a) Efficiency-corrected angular distribution as in Fig. 8 and (b) longitudinal-momentum distribution as in Fig. 6 but now for ground-state ${ }^{16} \mathrm{Ne}$ residuals produced in the neutron knockout reaction from the $E / A=58.2-\mathrm{MeV}^{17} \mathrm{Ne}$ beam.

ity to the transverse-momentum distribution assumed in the simulations and no extrapolation to small angles is needed. Thus the detection efficiency was significant higher; $36(5) \%$ compared to $3.98(64) \%$ for the same case with the HiRA detector. Similar corrections were made for particles that did not fall in their $E-\Delta E$ gate. The number of beam particles was counted directly in the same detector so acquisition dead-time corrections were not needed. Contributions to the systematic error were from the target thickness $(2 \%)$, efficiency $(11 \%)$ and the number of projectiles (5\%). In addition, as only 75 ground-state events were detected, there is also an $8 \%$ statistical error. The extracted cross section of $1.5(5)(3) \mathrm{mb}$ [(systematic) (statistical)] for the ${ }^{12} \mathrm{O}_{0^{+} ; 2}^{\text {g.s. }}$ is consistent with the value of $1.35(44) \mathrm{mb}$ obtained at the higher energy $(E / A=65.5 \mathrm{MeV})$. Therefore, the cross section for the higher bombarding energy obtained with the HiRA is not unusual and confirms that there is only modest dependence of the knockout cross sections on beam energy.

\section{THEORETICAL CROSS SECTIONS}

To allow comparison with the bound-final-states systematics, the $\sigma_{s p}$ were calculated with the same proce- 
dures as used in that work. Details can be found in [2]. Except for the ${ }^{9} \mathrm{C}$ beam cases, the residual-target optical potentials, for determination of their elastic scattering $S$-matrices, were constrained using the residues' neutron and proton densities obtained from Hartree-Fock calculations. For the $A=8$ residues, a Gaussian density of rms radius $2.38 \mathrm{fm}$ was used, a value consistent with high-energy reaction cross section data [36]. The overlap functions, specifically, the geometry of the Woods-Saxon binding potentials used to calculate the radial formfactors of the knocked-out nucleon, were also constrained by the rms radii of the different single-particle orbitals as obtained in the corresponding Hartree-Fock calculations for the projectile, see Ref. [2].

The shapes of the longitudinal and transverse momentum distributions are calculated for the dominant inelastic (stripping) mechanism following the formalism of [37]. In the case of the longitudinal distributions, such calculations have been compared to those from both continuumdiscretized coupled-channels calculations [38] and from eikonal calculations of the elastic (diffractive) component and found to be very similar. Predicted longitudinalmomentum distributions (red dashed curves) are compared to their experimental counterparts in Figs. 6, 12 and 15(b). These predictions have the experimental resolution incorporated via the Monte Carlo simulations and have been normalized to the same peak height as their experimental counterparts. Generally, the theoretical predictions explain the high-momentum edge of the observed distributions which are wider and have more significant lower-momentum tails. $P_{\|}$distributions for a number of these states were also calculated in the transfer-to-thecontinuum (TC) model, by Bonaccorso [39], for which momentum and energy sharing between the removed nucleon, residual, and target (in its ground state) is treated. In that model, the calculated high and low-momentum tails are no longer symmetric about the center value as is inherent in the eikonal-model predictions. However, the TC distributions do not explain the extent of the lowmomentum tails observed in the data, the experimental distributions being also wider than these predictions. Such low-momentum tails are commonly observed in knockout reactions to particle-bound final states [38, 4043]. Theoretically they have been attributed to either the dynamics of the removed nucleon inside the potential of the residual nucleus $[42,44]$ or caused by (a) the dissipative nature of the dominant inelastic removal (stripping) knockout mechanism, and (b) the energy and momentum transfer to the target in the kinematics of the elastic break-up mechanism [38]. Possibly the low-momentum tails in the $P_{\|}$distributions are linked to the "tail" components in the fitted angular distribution in Figs. 8, 13, and Fig. 15(a). It is also possible that these experimental distributions are biased, due to the angular acceptance of the detector, which has a greater efficiency for the "tail" component in the angular distribution.

Shell-model spectroscopic factors $C^{2} S$ for the required overlaps were calculated with the OXBASH code [45] and are listed in Tables I and II where the center-of-mass cor- rection from Eq. (1) has been included. For the ${ }^{9} \mathrm{C}$ projectile, the Cohen-Kurath effective interaction (POT) in the $p$ shell [46] was used while for the ${ }^{13} \mathrm{O}$ and ${ }^{17} \mathrm{Ne}$ projectiles the PSDWBT effective interactions in the $p s d$ space [47] was used to be more consistent with the calculations used in the bound-states systematics studies. For the two heavier projectiles we also considered the PSDMWK effective interaction [48] for comparison. Generally, the predicted spectroscopic factors are quite similar from the two psd interactions (within 20\%), except for the $2_{2}^{+} ; 2$ states in ${ }^{12} \mathrm{~N}$ and ${ }^{12} \mathrm{O}$ and the $2^{+} ; 2$ state in ${ }^{16} \mathrm{Ne}$ where the spectroscopic factors differ by $200 \%$ to $1000 \%$. Possibly the corresponding $2^{+}$levels have a different energy ordering with the two effective interactions. We note that Fortune argued, based on his spectroscopic factors, that the fourth $2^{+}$state should be strongly populated in a neutron-knockout reaction from ${ }^{13} \mathrm{O}$ and the second and third $2^{+}$states should be populated very weakly [49]. In view of these difficulties in assigning the appropriate $2^{+}$ state, we will ignore these three states in the subsequent discussions.

For the ${ }^{9} \mathrm{C}$ projectile, we also considered spectroscopic factors from the ab initio Variational Monte Carlo (VMC) model [50, 51], listed in the Table I. The VMC calculates the particle-unstable states in a bound-state approximation and thus the overlap functions fall exponentially at large separations between the core and the removed nucleon. However, the VMC overlap functions do incorporate effects beyond the shell model. For instance the VMC wavefunctions spread out with increasing excitation energy, for example, the ${ }^{8} \mathrm{~B}$ proton (neutron) rms radius increases from $2.45 \mathrm{fm}(2.14 \mathrm{fm})$ for ${ }^{8} \mathrm{~B}_{2^{+}}^{\text {g.s. }}$ to $2.77 \mathrm{fm}(2.41 \mathrm{fm})$ for the $0^{+} ; 2$ isobaric analog state. In addition removing a neutron from ${ }^{9} \mathrm{C}^{\text {g.s. }}$ to form ${ }^{8} \mathrm{C}^{\text {g.s. }}$ increases the proton rms radius from 2.47 to $2.88 \mathrm{fm}$. With the VMC spectroscopic factors, the single-particle cross sections were recomputed, using Woods-Saxon boundstate best fits to the VMC radial overlap functions, in the manner of Ref. [52].

\section{DISCUSSION}

The ratios, $\sigma_{\text {exp }} / \sigma_{t h}$ obtained for the exclusive, unbound final-state cross sections will be designated $R_{u}$ to distinguish these from the inclusive bound-states ratio. These are plotted against the separation energy $E_{s e p}$ of the removed nucleon in Fig. 16(a) for the ${ }^{9} \mathrm{C}$-projectile data. The error bars here only include systematic uncertainties which are not common to all channels so as to concentrate on their relative differences. We observe a rapid drop in the ratio with increasing $E_{\text {sep }}$. For neutron knockout to the unbound ground state of ${ }^{8} \mathrm{C}$ (square points), the results lie on the systematic trend observed for the proton-knockout results (circular points). This behavior is observed with both the shell-model (filled symbols) and VMC (open symbols) overlaps. Indeed the results obtained with the two structure models are quite similar; the lower spectroscopic factors predicted by the 


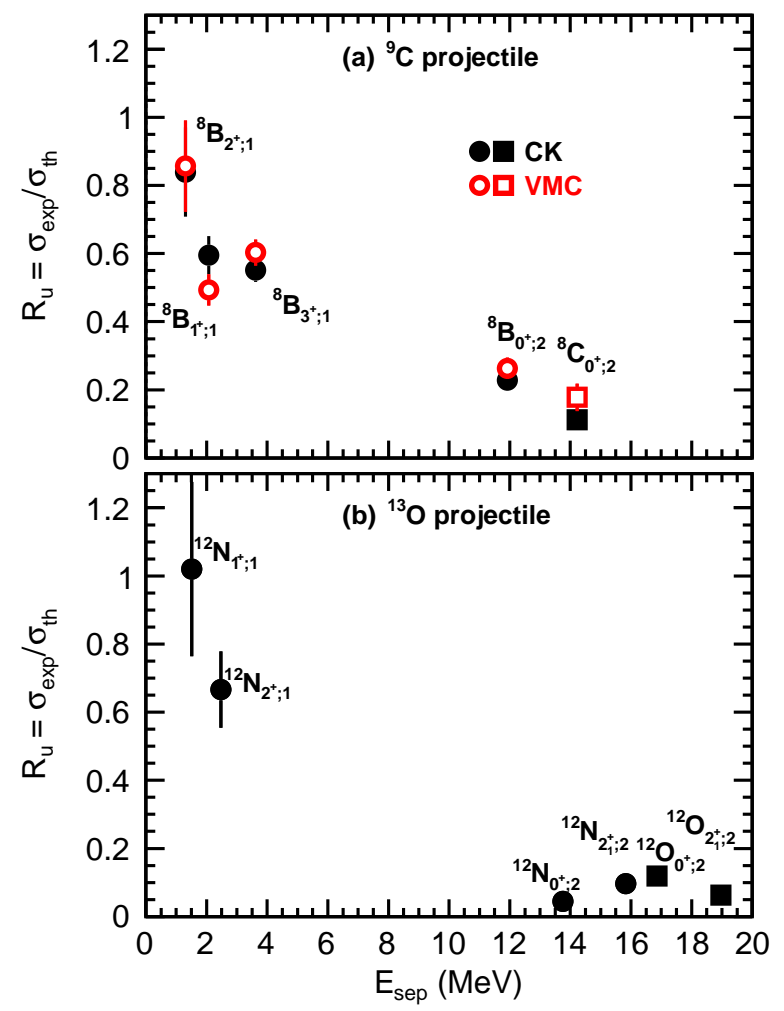

FIG. 16. Ratios of the experimental to theoretical cross sections for single-nucleon knockout channels as a function of the separation energy of the removed nucleon. (a) shows the results for the $E / A=66.8-\mathrm{MeV}^{9} \mathrm{C}$ beam with theoretical cross sections calculated with spectroscopic factors obtained with the Cohen-Kurath (CK) effective interaction in the shell model and with the Variational Monte Carlo (VMC) model. (b) results for the $E / A=65.4-\mathrm{MeV}^{13} \mathrm{O}$ beam. To concentrate on the relative difference between the channels, systematic uncertainties that are common to all channels are excluded from the error bars in both panels. Proton knockout channels are indicated with the circular data points, while neutron channels are indicated with the square data points.

VMC model are compensated by higher values predicted for $\sigma_{s p}$, a result of the $\mathrm{VMC}$ overlaps being more spatially extended.

The ${ }^{8} \mathrm{~B}_{2+}^{\text {g.s }}$ yield has the largest uncertainty due to the extrapolation of the experimental angular distributions. Due to the normalization of the $\sigma_{\exp }$ based on a previous measurement for this state, a decrease in the measured number of ${ }^{8} \mathrm{~B}_{2^{+}}^{g . s}$ fragments produced in the experiment would increase the deduced cross sections for the other states. A model-independent lower limit to this number is obtained by taking an extrapolation from the minimum-angle data point that goes linearly to zero at $0^{\circ}$ in Fig. 8(a). This decreases the yield by $23 \%$ and thus would increase the deduced cross sections for the other channels by the same amount. The general trend with $E_{\text {sep }}$ is similar, however now there is a unrealistic discontinuity in the slope of the ground-state angular distribution.

The ratios obtained for the ${ }^{13} \mathrm{O}$ projectile are displayed in Fig. 16(b) where again the systematic uncertainties which are common to all channels are excluded from the error bars. For the ${ }^{12} \mathrm{~N}_{1+; 1}^{\text {g.s. }}$ residuals which corresponds to $\Delta S=-15.36 \mathrm{MeV}$, the inclusive bound-state systematics would predict a value of $\sigma_{\text {exp }} / \sigma_{t h}$ of $0.8(1)$. Our extracted value of 1.0(4) is consistent with this. There is, again, a rapid drop in the ratio $R_{u}$ for the states with increasing $E_{\text {sep }}$ with similar values for states formed via neutron (circular points) and proton (square points) knockout reactions. The lowest ratio of $R_{u}=0.044(9)$ was obtained for the production of the IAS in ${ }^{12} \mathrm{~N}\left(0^{+} ; 2\right)$, however, it was suggested in Ref. [13] that this state may have a significant $\gamma$-decay branch in addition to the observed $2 p$ decay channel. If this is the case, the cross section and ratio should be larger than reported here. However, apart from this uncertainty, the ratios obtained with both the ${ }^{9} \mathrm{C}$ and ${ }^{13} \mathrm{O}$ projectiles show very similar dependencies on $E_{\text {sep }}$.

For the ${ }^{13} \mathrm{O}$ beam, proton knockout to the ground state of ${ }^{12} \mathrm{~N}$ corresponds to $\Delta S=-15.36 \mathrm{MeV}$ while neutron knockout to the ground state of ${ }^{12} \mathrm{O}$ is $15.36 \mathrm{MeV}$. So, based on the bound states, $R_{s}$, systematics (Fig. 1) we would expect a reduction of the ratio for ${ }^{12} \mathrm{O}_{0^{+} ; 2}^{g . s .}$ compared to ${ }^{12} \mathrm{~N}_{1^{+} ; 1}^{\text {g.s. }}$, as observed. One can make a similar argument for a reduction of the ${ }^{8} \mathrm{C}^{\text {g.s. }}$ ratio compared to the ${ }^{8} \mathrm{~B}^{\text {g.s }}$ ratio. However, the reduction of the ratio for these neutron-knockout channels is larger than expected. This is demonstrated in Fig. 1 which compares these exclusive $R_{u}$ ratios for ${ }^{8} \mathrm{C}_{0^{+} ; 2}^{\text {g.s. }}$ and ${ }^{12} \mathrm{O}_{0^{+} ; 2}^{\text {g.s. }}$ residuals to the $R_{s}$ systematics. Here we have included the full uncertainties associated with obtaining the experimental cross sections and, to be consistent, we use the ratio obtained with the shell-model inputs for the ${ }^{8} \mathrm{C}^{g . s}$ point. The ratios for ${ }^{12} \mathrm{O}^{\text {g.s. }}$ and ${ }^{8} \mathrm{C}^{\text {g.s. }}$ are around a factor of two to three smaller than the $R_{s}$ systematics for the same values of $\Delta S$. This large difference cannot be explained by uncertainties in normalization and the angular and momentum extrapolations employed. These two cases of knockout to (exclusive) resonance ground states thus show a further reduction in cross section than would be predicted from the inclusive, bound-states systematics. The result for neutron knockout from ${ }^{17} \mathrm{Ne}$ to the ground state of ${ }^{16} \mathrm{Ne}$ is also shown. It lies between the earlier systematics and our results for ${ }^{8} \mathrm{C}^{\text {g.s. }}$ and ${ }^{12} \mathrm{O}^{\text {g.s. }}$, possibly indicating a mass dependence.

The neutron knockout cross section from ${ }^{10} \mathrm{C}$ to the ${ }^{9} \mathrm{C}^{g . s}$. was measured by Grinyer et al. [52] giving a value of $R_{s}=0.48(2)$ with the theoretical cross section calculated with the Cohen-Kurath shell-model spectroscopic factor in the same manner as this work. As ${ }^{9} \mathrm{C}$ has no particle-bound excited states, the measured yield in this case is exclusive. We observe that, with a ${ }^{9} \mathrm{C}$ projectile containing one less neutron, the ratio for knockout to the unbound ${ }^{8} \mathrm{C}$ ground state has dropped to $0.12(6)$. Note, we are seeing a reduction in the ratio even though $\Delta S$ decreases by $\approx 4 \mathrm{MeV}$ between neutron knockout from ${ }^{10} \mathrm{C}$ and ${ }^{9} \mathrm{C}$.

We find a similar drop in the ratio for oxygen isotopes. Flavigny et al. measured the single-neutron 
knockout cross section from ${ }^{14} \mathrm{O}$ to ${ }^{13} \mathrm{O}^{\text {g.s. }}$ and obtained $\sigma_{\text {exp }} / \sigma_{t h}=0.26(2)[42]$. Again, the ${ }^{13} \mathrm{O}$ residual has no particle-bound excited states, so this ratio is for the exclusive cross section. For ${ }^{13} \mathrm{O}$, with one less neutron, the ratio for neutron knockout to ${ }^{12} \mathrm{O}^{\text {g.s. }}$ falls to $0.12(4)$. Thus, for both the carbon and oxygen isotopes, the ratio falls as the knockout reaction moves the residue across the proton drip line. This may portend a more general reduction in cross sections for knockout across drip lines, but it may also be peculiar to the three cases shown in Fig. 16.

If this drop in the ratio as one crosses the drip line is due to nuclear structure effects, it suggests that the structure of oxygen and carbon isotopes change dramatically as one crosses the proton drip line. The shell-model effective interactions used were constrained from nuclei closer to $\beta$ stability, so, using them at and beyond the drip lines may be problematic. However, we see that the ratio obtained with the VMC model for ${ }^{9} \mathrm{C} \rightarrow{ }^{8} \mathrm{C}$ was quite similar. For both ${ }^{13} \mathrm{O}$ and ${ }^{12} \mathrm{O}$, the ground-state configurations are predominately $p$-shell in the shell-model calculations. Close to the drip line we expect a quenching of the $Z=8$ shell gap due to the intruder $s_{1 / 2}$ orbital based on results obtained for the mirror nuclei ${ }^{13} \mathrm{~B}$ and ${ }^{12} \mathrm{Be}$. There is evidence that both nuclei have sizable $s d$-shell strength [53-55]. However, Fortune estimates the spectroscopic factor for proton removal from ${ }^{13} \mathrm{~B}$ is reduced only by $14 \%$ compared to the value calculated with just $p$-shell configurations [54]. Such a reduction is too small to account for the large fall in $\sigma_{\text {exp }} / \sigma_{t h}$ seen for ${ }^{13} \mathrm{O} \rightarrow{ }^{12} \mathrm{O}$.

In the ${ }^{13} \mathrm{~B} \rightarrow{ }^{12} \mathrm{Be}$ mirror proton knockout reaction, the inclusive bound-states yield was measured as $8.9(3) \mathrm{mb}$ with a $E / A \approx 400-\mathrm{MeV}$ beam and a carbon target [56]. The inclusive yield can have contributions from the $0_{1}^{+}$ ground state and the $0_{2}^{+}, 2_{1}^{+}$, and $1_{1}^{-}$excited states, all located below the neutron separation energy. However, based on the shell-model spectroscopic factors, or those from Fortune [54], the $0_{1}^{+}$and $2_{1}^{+}$contributions are expected to be the most substantial. We calculate $R_{s}=0.35$ (1) from this inclusive yield which is consistent with the $R_{s}$ systematics for the relevant $\Delta S=12.15 \mathrm{MeV}$. For the ${ }^{13} \mathrm{O} \rightarrow{ }^{12} \mathrm{O}$ case, the sum of the $0_{1}^{+}$and $2_{1}^{+}$contributions is $3.6(10) \mathrm{mb}$. If the $0_{2}^{+}$and $1_{1}^{-}$states are present, they will contribute to the shoulder on the low-energy side of the observed $2_{1}^{+}$peak in the invariant-mass spectrum of Fig. 10(c), for which a separate peak was introduced in the fit to the data [13]. But, this shoulder could also be associated with the $2_{2}^{+}$state if it has a $2 p$-decay branch to the first excited state in ${ }^{10} \mathrm{C}[13]$ (dashed arrow in Fig. 9). Based on this, we include the cross section for the fitted shoulder peak in Fig. 10(c) with those from the $0_{1}^{+}$and $2_{1}^{+}$peaks to give a maximum cross section for the four states of $4.0(10) \mathrm{mb}$. This is still a factor of $0.44(12)$ less than the inclusive yield for the corresponding states in the mirror reaction. Of course the target and beam energy were different in these two mirror reactions, but the difference in cross section between a $\mathrm{C}$ and a Be target is quite minor. Ref. [52] finds an $\approx 15 \%$ increase in the cross section for a $\mathrm{C}$ target. On the other hand, the higher beam energy should slightly reduce the cross section compensating for the increase due to the $\mathrm{C}$ target. In the eikonal model, the corresponding $\sigma_{\text {s.p. }}$ values differ by only $2-3 \%$ in these mirror reactions. Thus, these differences in cross sections indicate an observed breaking of mirror symmetry and further emphasizes the low values of the neutron knockout cross sections measured in this work.

As the drip-line is approached and crossed, the coupling of the shell-model configurations to the continuum should be considered. Neither the shell model nor the VMC model include this effect. The effect of continuum coupling on spectroscopic factors has been considered for particle-bound systems. These studies predicted that spectroscopic factors are reduced by $15 \%$ or less [57]. The continuum is of course expected to break mirror symmetry between the particle-unstable ${ }^{12} \mathrm{O}$ and its particlestable mirror ${ }^{12} \mathrm{Be}$. Grigorenko et al. predicted a tens of percent increase in occupancy of the $\nu\left(s_{1 / 2}\right)^{2}$ configuration in ${ }^{12} \mathrm{O}$ compared to the corresponding $\pi\left(s_{1 / 2}\right)^{2}$ configuration in ${ }^{12} \mathrm{~B}$ due to the continuum, which they ascribed to a three-body Thomas-Ehrman effect [58]. Although this would modify the ${ }^{13} \mathrm{O} \rightarrow{ }^{12} \mathrm{O}$ spectroscopic factor, the effect does not appear sufficient to produce the large changes in $\sigma_{e x p} / \sigma_{t h}$ observed for the oxygen isotopes and the mirror reaction.

Significant changes in nuclear structure are expected for near-threshold [59-61] states and those close to exceptional points [62]. Near-threshold states are located close in energy to a threshold for a particular exit channel and the coupling to this continuum aligns the wave function to this exit-channel configuration. The ${ }^{16} \mathrm{Ne}$ ground state, one of the residuals considered in this work, has been discussed as such a near-threshold state [59] which, based on the most recent measurement of its energy [14], is located $196(20) \mathrm{keV}$ above the $p+{ }^{15} \mathrm{~F}_{1 / 2^{+}}^{g . s}$ threshold. Due to Coulomb effects, the alignment of the wave function is strongest if the state is located above the proton threshold with the optimum value at $0.5 \mathrm{MeV}$ above the threshold for ${ }^{16} \mathrm{Ne}$ [59]. Based on the most recent measurements [13], the ${ }^{12} \mathrm{O}^{\text {g.s. }}$ is located $0.660 \mathrm{MeV}$ above the $p+{ }^{11} \mathrm{~N}_{1 / 2^{+}}^{g . s .}$ threshold so it might also have a nearthreshold configuration. Indeed, a preliminary calculation with the Shell Model Embedded in the Continuum [63] suggests that the inclusion of continuum coupling for the ${ }^{13} \mathrm{O} \rightarrow{ }^{12} \mathrm{O}^{g . s}$ case does lead to a significant suppression of its spectroscopic factor [64]. In addition, preliminary calculations within a different theoretical framework, the Gamow Shell model [65, 66], suggest a similar suppression of the ${ }^{9} \mathrm{C} \rightarrow{ }^{8} \mathrm{C}^{\text {g.s }}$ spectroscopic factor [67].

Clearly further theoretical work is needed to extend such studies to other unbound resonances. However, not all of the resonance states studied in this work can be considered near-threshold states or close to exceptional points and it is not at all clear why a systematic trend with $E_{\text {sep }}$ would be produced by the continuum coupling effect. One should also note that, for knockout to a particle-unstable state, there is not a unique spectro- 
scopic factor but a distribution of strength. Indeed the reaction and structure should be considered in a consistent and time dependent model that accounts for the possible interference between the resonant and non-resonant continuum [68, 69].

We are aware of one other study where a cross section for knockout to a resonance state were measured. Peters et al. looked at single-neutron knockout from ${ }^{12} \mathrm{Be}$ projectiles and observed the $E^{*}=3.949 \mathrm{MeV}, J^{\pi}=3 / 2_{2}^{+}$ state in the $n+{ }^{10} \mathrm{Be}$ invariant-mass spectrum [70]. The production of this state corresponds to the removal of a neutron from ${ }^{12} \mathrm{Be}$ with a separation energy of $E_{\text {sep }}=7.1$ $\mathrm{MeV}$. For the relevant value of $\Delta S$ for ${ }^{12} \mathrm{Be}$, the $R_{s}$ systematics suggest that $\sigma_{\text {exp }} / \sigma_{t h} \approx 1$. Indeed, the measured cross section was consistent with the shell/eikonal model calculations, so this indicates that not all cases of knockout to individual resonances give rise to $\sigma_{\text {exp }} / \sigma_{t h}$ values much smaller than the bound-states systematics.

Perhaps the role of the continuum coupling on the structure of the residuals is not the only physics driver here. The projectiles considered in this work are all fragile with small proton separation energies and low Coulomb barriers. For the residuals with the small $R_{u}$ values in this work, one is attempting to remove a wellbound nucleon. The eikonal model assumes that one does so with a minimal disruption of the remaining nucleons, since the residue target interaction is computed from the $\mathrm{HF}$ ground-state density of the residue for all final states. In addition, the measured longitudinal-momentum distributions show substantial low-momentum tails as is common in other knockout studies suggesting the importance of dissipative processes that may suggest missing dynamical effects may play a role in explaining the observed trends.

\section{CONCLUSION}

Cross sections for single-nucleon knockout from the light proton-drip-line projectiles ${ }^{9} \mathrm{C},{ }^{13} \mathrm{O}$, and ${ }^{17} \mathrm{Ne}$ have been extracted from previously-published data sets. Most of the residuals produced in the knockout reactions are resonances and were detected from the invariant mass of their decay products. Corresponding theoretical cross sections were calculated using the eikonal model and spectroscopic factors from the shell model. All other inputs were consistent with those used in the systematic studies of inclusive yields for particle-bound residuals $[2,3]$. For the ${ }^{9} \mathrm{C}$ residuals, spectroscopic factors and overlap functions from the Variational Monte Carlo model were also considered and found to give similar calculated cross sections.

The ratio of the experimental to theoretical cross section for these exclusive, unbound states, $R_{u}$, was found to drop rapidly as more deeply-bound nucleons were removed. Proton and neutron knockout channels followed the same trend within the experimental uncertainties. $R_{u}$ for neutron knockout to the ground-state residuals was found to be significantly smaller than the $R_{s}$ interpolated from the bound-state systematics at the same value of separation-energy asymmetry, $\Delta S$. The physical origin of these observations is not totally clear. As the residuals associated with knockout of deeply-bound nucleons are resonances, modifications to their nuclear structure due to coupling of the shell-model-like configurations to the continuum will play a role especially for knockout to near-threshold states, but we do not know whether this mechanism can explain all of the observed trends.

Clearly more theoretical and experiment studies are needed to understand these observations. Our experimental studies are confined to light proton-rich projectiles with the same $N-Z$ values. It would interesting to see if similar trends would be observed for heavier projectiles and also for projectiles at the neutron-drip line. Finally, if one wants to use knockout reactions to cross one of the drip lines to create such resonances, then one should use caution in making cross-section estimates based on the $R_{s}$ systematics observed for inclusive cross sections to bound residuals.

\section{ACKNOWLEDGMENTS}

The authors would like to thank Marek Płoszajzcak and Robert Wiringa for helpful discussions. This material is based upon work supported by the U.S. Department of Energy, Office of Science, Office of Nuclear Physics under award number DE-FG02-87ER-40316. JAT acknowledges support from the Science and Technology Facilities Council (U.K.) Grant No. ST/L005743/1.
[1] P.G. Hansen and J.A. Tostevin, "Direct reactions with exotic nuclei," Annu. Rev. Nucl. Part. Sci. 53, 219-261 (2003), https://doi.org/10.1146/annurev.nucl.53.041002.110406.

[2] A. Gade, P. Adrich, D. Bazin, M. D. Bowen, B. A. Brown, C. M. Campbell, J. M. Cook, T. Glasmacher, P. G. Hansen, K. Hosier, S. McDaniel, D. McGlinchery, A. Obertelli, K. Siwek, L. A. Riley, J. A. Tostevin, and D. Weisshaar, "Reduction of spectroscopic strength: Weakly-bound and strongly-bound single-particle states studied using one-nucleon knockout reactions," Phys. Rev. C 77, 044306 (2008).
[3] J. A. Tostevin and A. Gade, "Systematics of intermediate-energy single-nucleon removal cross sections," Phys. Rev. C 90, 057602 (2014).

[4] A. Gade, D. Bazin, B. A. Brown, C. M. Campbell, J. A. Church, D. C. Dinca, J. Enders, T. Glasmacher, P. G. Hansen, Z. Hu, K. W. Kemper, W. F. Mueller, H. Olliver, B. C. Perry, L. A. Riley, B. T. Roeder, B. M. Sherrill, J. R. Terry, J. A. Tostevin, and K. L. Yurkewicz, "Reduced occupancy of the deeply bound $0 d_{5 / 2}$ neutron state in ${ }^{32}$ Ar," Phys. Rev. Lett. 93, 042501 (2004).

[5] J. Daz-Corts, J. Benlliure, J. L. Rodrguez-Snchez, 
H. lvarez Pol, T. Aumann, C. A. Bertulani, B. Blank, E. Casarejos, D. Cortina-Gil, D. Dragosavac, V. Fhr, A. Gargano, M. Gascn, W. Gawlikowicz, A. Heinz, K. Helariutta, A. Kelic-Heil, S. Lukic, F. Montes, D. Prez-Loureiro, L. Pienkowski, K-H. Schmidt, M. Staniou, K. Subotic, K. Smmerer, J. Taieb, and A. Trzcinska, "Systematic reduction of the proton-removal cross section in neutron-rich medium-mass nuclei," (2020), arXiv:2006.05774 [nucl-ex].

[6] Jenny Lee, M.B. Tsang, D. Bazin, D. Coupland, V. Henzl, D. Henzlova, M. Kilburn, W. G. Lynch, A. M. Rogers, A. Sanetullaev, Z. Y. Sun, M. Youngs, R. J. Charity, L. G. Sobotka, M. Famiano, S. Hudan, D. Shapira, P. O'Malley, W. A. Peters, K. Y. Chae, and K. Schmitt, "Neutron spectroscopic factors of ${ }^{34} \mathrm{Ar}$ and ${ }^{46} \mathrm{Ar}$ from $(p, d)$ transfer reactions," Phys. Rev. C 83, 014606 (2011).

[7] F. Flavigny, A. Gillibert, L. Nalpas, A. Obertelli, N. Keeley, C. Barbieri, D. Beaumel, S. Boissinot, G. Burgunder, A. Cipollone, A. Corsi, J. Gibelin, S. Giron, J. Guillot, F. Hammache, V. Lapoux, A. Matta, E. C. Pollacco, R. Raabe, M. Rejmund, N. de Séreville, A. Shrivastava, A. Signoracci, and Y. Utsuno, "Limited asymmetry dependence of correlations from single nucleon transfer," Phys. Rev. Lett. 110, 122503 (2013).

[8] B. P. Kay, J. P. Schiffer, and S. J. Freeman, "Quenching of cross sections in nucleon transfer reactions," Phys. Rev. Lett. 111, 042502 (2013).

[9] L. Atar, S. Paschalis, C. Barbieri, C. A. Bertulani, P. Díaz Fernández, M. Holl, M. A. Najafi, V. Panin, H. Alvarez-Pol, and et al. Aumann, "Quasifree $(p, 2 p)$ reactions on oxygen isotopes: Observation of isospin independence of the reduced single-particle strength," Phys. Rev. Lett. 120, 052501 (2018).

[10] S. Kawase, T. Uesaka, T. L. Tang, D. Beaumel, M. Dozono, T. Fukunaga, T. Fujii, N. Fukuda, A. GalindoUribarri, S. Hwang, N. Inabe, T. Kawabata, T. Kawahara, W. Kim, K. Kisamori, M. Kobayashi, T. Kubo, Y. Kubota, K. Kusaka, C. Lee, Y. Maeda, H. Matsubara, S. Michimasa, H. Miya, T. Noro, Y. Nozawa, A. Obertelli, K. Ogata, S. Ota, E. Padilla-Rodal, S. Sakaguchi, H. Sakai, M. Sasano, S. Shimoura, S. Stepanyan, H. Suzuki, T. Suzuki, M. Takaki, H. Takeda, A. Tamii, H. Tokieda, T. Wakasa, T. Wakui, K. Yako, J. Yasuda, Y. Yanagisawa, R. Yokoyama, K. Yoshida, K. Yoshida, and J. Zenihiro, "Exclusive quasi-free proton knockout from oxygen isotopes at intermediate energies," Prog. Theor. Exp. Phys. 2018, $021 D 01$ (2018).

[11] M.S. Wallace, M.A. Famiano, M.-J. van Goethem, A.M. Rogers, W.G. Lynch, J. Clifford, F. Delaunay, J. Lee, S. Labostov, M. Mocko, L. Morris, A. Moroni, B.E. Nett, D.J. Oostdyk, R. Krishnasamy, M.B. Tsang, R.T. de Souza, S. Hudan, L.G. Sobotka, R.J. Charity, J. Elson, and G.L. Engel, "The high resolution array (HiRA) for rare isotope beam experiments," Nucl Instrum. Methods A 583, $302-312$ (2007).

[12] R. J. Charity, K. W. Brown, J. Elson, W. Reviol, L. G. Sobotka, W. W. Buhro, Z. Chajecki, W. G. Lynch, J. Manfredi, R. Shane, R. H. Showalter, M. B. Tsang, D. Weisshaar, J. Winkelbauer, S. Bedoor, D. G. McNeel, and A. H. Wuosmaa, "Invariant-mass spectroscopy of ${ }^{18} \mathrm{Ne},{ }^{16} \mathrm{O}$, and ${ }^{10} \mathrm{C}$ excited states formed in neutrontransfer reactions," Phys. Rev. C 99, 044304 (2019).

[13] T. B. Webb, R. J. Charity, J. M. Elson, D. E. M. Hoff, C. D. Pruitt, L. G. Sobotka, K. W. Brown, J. Barney,
G. Cerizza, J. Estee, G. Jhang, W. G. Lynch, J. Manfredi, P. Morfouace, C. Santamaria, S. Sweany, M. B. Tsang, T. Tsang, S. M. Wang, Y. Zhang, K. Zhu, S. A. Kuvin, D. McNeel, J. Smith, A. H. Wuosmaa, and Z. Chajecki, "Particle decays of levels in ${ }^{11,12} \mathrm{~N}$ and ${ }^{12} \mathrm{O}$ investigated with the invariant-mass method," Phys. Rev. C 100, 024306 (2019).

[14] K. W. Brown, R. J. Charity, L. G. Sobotka, Z. Chajecki, L. V. Grigorenko, I. A. Egorova, Yu. L. Parfenova, M. V. Zhukov, S. Bedoor, W. W. Buhro, J. M. Elson, W. G. Lynch, J. Manfredi, D. G. McNeel, W. Reviol, R. Shane, R. H. Showalter, M. B. Tsang, J. R. Winkelbauer, and A. H. Wuosmaa, "Observation of long-range three-body Coulomb effects in the decay of ${ }^{16} \mathrm{Ne}$," Phys. Rev. Lett. 113, 232501 (2014).

[15] K. W. Brown, R. J. Charity, L. G. Sobotka, L. V. Grigorenko, T. A. Golubkova, S. Bedoor, W. W. Buhro, Z. Chajecki, J. M. Elson, W. G. Lynch, J. Manfredi, D. G. McNeel, W. Reviol, R. Shane, R. H. Showalter, M. B. Tsang, J. R. Winkelbauer, and A. H. Wuosmaa, "Interplay between sequential and prompt two-proton decay from the first excited state of ${ }^{16}$ Ne," Phys. Rev. C 92, 034329 (2015).

[16] R. J. Charity, J. M. Elson, J. Manfredi, R. Shane, L. G. Sobotka, B. A. Brown, Z. Chajecki, D. Coupland, H. Iwasaki, M. Kilburn, Jenny Lee, W. G. Lynch, A. Sanetullaev, M. B. Tsang, J. Winkelbauer, M. Youngs, S. T. Marley, D. V. Shetty, A. H. Wuosmaa, T. K. Ghosh, and M. E. Howard, "Investigations of three-, four-, and five-particle decay channels of levels in light nuclei created using a ${ }^{9}$ C beam," Phys. Rev. C 84, 014320 (2011).

[17] I. A. Egorova, R. J. Charity, L. V. Grigorenko, Z. Chajecki, D. Coupland, J. M. Elson, T. K. Ghosh, M. E. Howard, H. Iwasaki, M. Kilburn, Jenny Lee, W. G. Lynch, J. Manfredi, S. T. Marley, A. Sanetullaev, R. Shane, D. V. Shetty, L. G. Sobotka, M. B. Tsang, J. Winkelbauer, A. H. Wuosmaa, M. Youngs, and M. V. Zhukov, "Democratic decay of ${ }^{6}$ Be exposed by correlations," Phys. Rev. Lett. 109, 202502 (2012).

[18] R. J. Charity, L. G. Sobotka, N. J. Robertson, D. G. Sarantites, J. Dinius, C. K. Gelbke, T. Glasmacher, D. O. Handzy, W. C. Hsi, M. J. Huang, W. G. Lynch, C. P. Montoya, G. F. Peaslee, C. Schwarz, and M. B. Tsang, "Prompt and sequential decay processes in the fragmentation of $40 \mathrm{MeV} /$ nucleon ${ }^{20}$ Ne projectiles," Phys. Rev. C 52, 3126-3150 (1995).

[19] P. Morfouace, W.G. Lynch, and M.B. Tsang, "Chargedparticle detection efficiencies of close-packed CsI arrays," Nucl. Instrum. Methods Phys. Res. A 848, 45 - 53 (2017).

[20] R. J. Charity, J. M. Elson, J. Manfredi, R. Shane, L. G. Sobotka, Z. Chajecki, D. Coupland, H. Iwasaki, M. Kilburn, Jenny Lee, W. G. Lynch, A. Sanetullaev, M. B. Tsang, J. Winkelbauer, M. Youngs, S. T. Marley, D. V. Shetty, A. H. Wuosmaa, T. K. Ghosh, and M. E. Howard, " $2 p$ - $2 p$ decay of ${ }^{8} \mathbf{C}$ and isospin-allowed $2 p$ decay of the isobaric-analog state in ${ }^{8} \mathbf{B}$," Phys. Rev. C 82, 041304(R) (2010).

[21] K. W. Brown, W. W. Buhro, R. J. Charity, J. M. Elson, W. Reviol, L. G. Sobotka, Z. Chajecki, W. G. Lynch, J. Manfredi, R. Shane, R. H. Showalter, M. B. Tsang, D. Weisshaar, J. R. Winkelbauer, S. Bedoor, and A. H. Wuosmaa, "Two-proton decay from the isobaric analog state in ${ }^{8}$ B," Phys. Rev. C 90, 027304 (2014).

[22] Evaluated Nuclear Structure Data File (ENSDF), 
http://www.nndc.bnl.gov/ensdf/.

[23] K. W. Brown, R. J. Charity, J. M. Elson, W. Reviol, L. G. Sobotka, W. W. Buhro, Z. Chajecki, W. G. Lynch, J. Manfredi, R. Shane, R. H. Showalter, M. B. Tsang, D. Weisshaar, J. R. Winkelbauer, S. Bedoor, and A. H. Wuosmaa, "Proton-decaying states in light nuclei and the first observation of ${ }^{17} \mathrm{Na}$," Phys. Rev. C 95, 044326 (2017).

[24] R. J. Charity, J. M. Elson, J. Manfredi, R. Shane, L. G. Sobotka, Z. Chajecki, D. Coupland, H. Iwasaki, M. Kilburn, Jenny Lee, W. G. Lynch, A. Sanetullaev, M. B. Tsang, J. Winkelbauer, M. Youngs, S. T. Marley, D. V. Shetty, and A. H. Wuosmaa, "Spin alignment of excited projectiles due to target spin-flip interactions," Phys. Rev. C 91, 024610 (2015).

[25] D. E. M. Hoff, R. J. Charity, K. W. Brown, C. D. Pruitt, L. G. Sobotka, T. B. Webb, G. Potel, B. Roeder, and A. Saastamoinen, "Large longitudinal spin alignment of excited projectiles in intermediate energy inelastic scattering," Phys. Rev. Lett. 119, 232501 (2017).

[26] D. E. M. Hoff, G. Potel, K. W. Brown, R. J. Charity, C. D. Pruitt, L. G. Sobotka, T. B. Webb, B. Roeder, and A. Saastamoinen, "Large longitudinal spin alignment generated in inelastic nuclear reactions," Phys. Rev. C 97, 054605 (2018).

[27] D. Bazin, R. J. Charity, R. T. de Souza, M. A. Famiano, A. Gade, V. Henzl, D. Henzlova, S. Hudan, J. Lee, S. Lukyanov, W. G. Lynch, S. McDaniel, M. Mocko, A. Obertelli, A. M. Rogers, L. G. Sobotka, J. R. Terry, J. A. Tostevin, M. B. Tsang, and M. S. Wallace, "Mechanisms in knockout reactions," Phys. Rev. Lett. 102, 232501 (2009).

[28] B. A. Brown, P. G. Hansen, B. M. Sherrill, and J. A. Tostevin, "Absolute spectroscopic factors from nuclear knockout reactions," Phys. Rev. C 65, 061601(R) (2002).

[29] J. Enders, T. Baumann, B. A. Brown, N. H. Frank, P. G. Hansen, P. R. Heckman, B. M. Sherrill, A. Stolz, M. Thoennessen, J. A. Tostevin, E. J. Tryggestad, S. Typel, and M. S. Wallace, "Spectroscopic factors measured in inclusive proton-knockout reactions on ${ }^{8} \mathrm{~B}$ and ${ }^{9} \mathrm{C}$ at intermediate energies," Phys. Rev. C 67, 064301 (2003).

[30] S T Wang, Y P Xu, and D Y Pang, "Energy dependence of the reduced single-particle strength for strongly-bound proton removal on ${ }^{16}$ C," Phys. Scr. 94, 015302 (2018).

[31] M. F. Jager, R. J. Charity, J. M. Elson, J. Manfredi, M. H. Mahzoon, L. G. Sobotka, M. McCleskey, R. G. Pizzone, B. T. Roeder, A. Spiridon, E. Simmons, L. Trache, and M. Kurokawa, "Two-proton decay of ${ }^{12} \mathrm{O}$ and its isobaric analog state in ${ }^{12} \mathrm{~N}$, " Phys. Rev. C 86, 011304(R) (2012).

[32] L. G. Sobotka, W. W. Buhro, R. J. Charity, J. M. Elson, M. F. Jager, J. Manfredi, M. H. Mahzoon, A. M. Mukhamedzhanov, V. Eremenko, M. McCleskey, R. G. Pizzone, B. T. Roeder, A. Spiridon, E. Simmons, L. Trache, M. Kurokawa, and P. Navrátil, "Proton decay of excited states in ${ }^{12} \mathrm{~N}$ and ${ }^{13} \mathrm{O}$ and the astrophysical ${ }^{11} \mathrm{C}(p, \gamma){ }^{12} \mathrm{~N}$ reaction rate," Phys. Rev. C 87, 054329 (2013).

[33] B. B. Skorodumov, G. V. Rogachev, P. Boutachkov, A. Aprahamian, V. Z. Goldberg, A. Mukhamedzhanov, S. Almaraz, H. Amro, F. D. Becchetti, S. Brown, Y. Chen, H. Jiang, J. J. Kolata, L. O. Lamm, M. Quinn, and A. Woehr, "Lowest excited states of ${ }^{13} \mathrm{O}$," Phys. Rev. C 75, 024607 (2007).
[34] R. J. Charity, K. W. Brown, J. Okołowicz, M. Płoszajczak, J. M. Elson, W. Reviol, L. G. Sobotka, W. W. Buhro, Z. Chajecki, W. G. Lynch, J. Manfredi, R. Shane, R. H. Showalter, M. B. Tsang, D. Weisshaar, J. R. Winkelbauer, S. Bedoor, and A. H. Wuosmaa, "Spin alignment following inelastic scattering of ${ }^{17} \mathrm{Ne}$, lifetime of ${ }^{16} \mathrm{~F}$, and its constraint on the continuum coupling strength," Phys. Rev. C 97, 054318 (2018).

[35] D. Bazin, V. Andreev, A. Becerril, M. Dolans, P.F. Mantica, J. Ottarson, H. Schatz, J.B. Stoker, and J. Vincent, "Radio frequency fragment separator at NSCL," Nucl. Instrum. Methods Phys. Res. A 606, 314 - 319 (2009).

[36] A. Ozawa, T. Suzuki, and I. Tanihata, "Nuclear size and related topics," Nucl. Phys. A 693, 32 - 62 (2001), radioactive Nuclear Beams.

[37] C. A. Bertulani and P. G. Hansen, "Momentum distributions in stripping reactions of radioactive projectiles at intermediate energies," Phys. Rev. C 70, 034609 (2004).

[38] J. A. Tostevin, D. Bazin, B. A. Brown, T. Glasmacher, P. G. Hansen, V. Maddalena, A. Navin, and B. M. Sherrill, "Single-neutron removal reactions from ${ }^{15} \mathrm{C}$ and ${ }^{11}$ Be: Deviations from the eikonal approximation," Phys. Rev. C 66, 024607 (2002).

[39] A. Bonaccorso, "Direct reaction theories for exotic nuclei: An introduction via semi-classical methods," Prog. Part. Nucl. Phys. 101, 1 (2018).

[40] A. Gade, D. Bazin, C. A. Bertulani, B. A. Brown, C. M. Campbell, J. A. Church, D. C. Dinca, J. Enders, T. Glasmacher, P. G. Hansen, Z. Hu, K. W. Kemper, W. F. Mueller, H. Olliver, B. C. Perry, L. A. Riley, B. T. Roeder, B. M. Sherrill, J. R. Terry, J. A. Tostevin, and K. L. Yurkewicz, "Knockout from ${ }^{46} \mathrm{Ar}: \ell=3$ neutron removal and deviations from eikonal theory," Phys. Rev. C 71, 051301(R) (2005).

[41] G. F. Grinyer, D. Bazin, A. Gade, J. A. Tostevin, P. Adrich, M. D. Bowen, B. A. Brown, C. M. Campbell, J. M. Cook, T. Glasmacher, S. McDaniel, P. Navrátil, A. Obertelli, S. Quaglioni, K. Siwek, J. R. Terry, D. Weisshaar, and R. B. Wiringa, "Knockout reactions from $p$ shell nuclei: Tests of ab initio structure models," Phys. Rev. Lett. 106, 162502 (2011).

[42] F. Flavigny, A. Obertelli, A. Bonaccorso, G. F. Grinyer, C. Louchart, L. Nalpas, and A. Signoracci, "Nonsudden limits of heavy-ion induced knockout reactions," Phys. Rev. Lett. 108, 252501 (2012).

[43] R. Shane, R. J. Charity, L. G. Sobotka, D. Bazin, B. A. Brown, A. Gade, G. F. Grinyer, S. McDaniel, A. Ratkiewicz, D. Weisshaar, A. Bonaccorso, and J. A. Tostevin, "Proton and neutron knockout from ${ }^{36} \mathrm{Ca}$," Phys. Rev. C 85, 064612 (2012).

[44] K. Ogata, K. Yoshida, and K. Minomo, "Asymmetry of the parallel momentum distribution of $(p, p n)$ reaction residues," Phys. Rev. C 92, 034616 (2015).

[45] B. A. Brown, A. Etchegoyen, N. S. Godwin, W. D. M. rae, W. A. Richter, W. E. Ormand, E. K. Warburton, J. S. Winfield, L. Zhao, and C. H. Zimmerman, Tech. Rep. MSU-NSCL-1289 (National SuperConducting Cyclotron Laboratory, Michigan State University, East Lansing Michigan, 2004).

[46] S. Cohen and D. Kurath, "Effective interactions for the 1p shell," Nucl. Phys. 73, 1 - 24 (1965).

[47] E. K. Warburton and B. A. Brown, "Effective interactions for the 0p1s0d nuclear shell-model space," Phys. Rev. C 46, 923-944 (1992).

[48] D.J. Millener and D. Kurath, "The particle-hole interac- 
tion and the beta decay of ${ }^{14} \mathrm{~B}, "$ Nucl. Phys. A 255, 315 -338 (1975).

[49] H T Fortune, "Splitting of strengths in neutron removal from ${ }^{13}$ O," J. Phys. G 43, 115102 (2016).

[50] I. Brida, Steven C. Pieper, and R. B. Wiringa, "Quantum Monte Carlo calculations of spectroscopic overlaps in $A \leqslant 7$ nuclei," Phys. Rev. C 84, 024319 (2011).

[51] R. Wirenga, "VMC spectroscopic overlaps," https://www.phy.anl.gov/theory/research/overlaps/.

[52] G. F. Grinyer, D. Bazin, A. Gade, J. A. Tostevin, P. Adrich, M. D. Bowen, B. A. Brown, C. M. Campbell, J. M. Cook, T. Glasmacher, S. McDaniel, A. Obertelli, K. Siwek, J. R. Terry, D. Weisshaar, and R. B. Wiringa, "Systematic study of $p$-shell nuclei via single-nucleon knockout reactions," Phys. Rev. C 86, 024315 (2012).

[53] N. Aoi, K. Yoneda, E. Ideguchi, T. Kishida, T. Nakamura, M. Notani, H. Sakurai, T. Teranishi, Y. Watanabe, H. Wu, A. Yoshida, H. Miyatake, Y. Yamamoto, H. Ogawa, S. S. Yamamoto, and M. Ishihara, "Anomaly of $N=8$ shell closure in neutron-rich Be and B isotopes studied via delayed neutron emitting ${ }^{14} \mathrm{Be} \beta$ decay," Phys. Rev. C 66, 014301 (2002).

[54] H.T. Fortune, "Population of bound and unbound states of ${ }^{12}$ Be in proton removal from ${ }^{13} \mathrm{~B}$," Phys. Lett. B $\mathbf{7 5 5}$, $351-352$ (2016).

[55] A. Krieger, K. Blaum, M. L. Bissell, N. Frömmgen, Ch. Geppert, M. Hammen, K. Kreim, M. Kowalska, J. Krämer, T. Neff, R. Neugart, G. Neyens, W. Nörtershäuser, Ch. Novotny, R. Sánchez, and D. T. Yordanov, "Nuclear charge radius of ${ }^{12}$ Be," Phys. Rev. Lett. 108, 142501 (2012).

[56] R. Thies, A. Heinz, T. Adachi, Y. Aksyutina, J. Alcantara-Núñes, S. Altstadt, H. Alvarez-Pol, N. Ashwood, T. Aumann, V. Avdeichikov, M. Barr, S. BeceiroNovo, D. Bemmerer, J. Benlliure, C. A. Bertulani, K. Boretzky, M. J. G. Borge, G. Burgunder, M. Camaño, C. Caesar, E. Casarejos, W. Catford, J. Cederkäll, S. Chakraborty, M. Chartier, L. V. Chulkov, D. CortinaGil, R. Crespo, U. Datta, P. Díaz Fernández, I. Dillmann, Z. Elekes, J. Enders, O. Ershova, A. Estradé, F. Farinon, L. M. Fraile, M. Freer, M. Freudenberger, H. O. U. Fynbo, D. Galaviz, H. Geissel, R. Gernhäuser, K. Göbel, P. Golubev, D. Gonzalez Diaz, J. Hagdahl, T. Heftrich, M. Heil, M. Heine, A. Henriques, M. Holl, G. Ickert, A. Ignatov, B. Jakobsson, H. T. Johansson, B. Jonson, N. Kalantar-Nayestanaki, R. Kanungo, R. Knöbel, T. Kröll, R. Krücken, J. Kurcewicz, N. Kurz, M. Labiche, C. Langer, T. Le Bleis, R. Lemmon, O. Lepyoshkina, S. Lindberg, J. Machado, J. Marganiec, V. Maroussov, M. Mostazo, A. Movsesyan, A. Najafi, T. Nilsson, C. Nociforo, V. Panin, S. Paschalis, A. Perea, M. Petri, S. Pietri, R. Plag, A. Prochazka, A. Rahaman, G. Rastrepina, R. Reifarth, G. Ribeiro, M. V. Ricciardi, C. Rigollet, K. Riisager, M. Röder, D. Rossi, J. Sanchez del Rio, D. Savran, H. Scheit, H. Simon, O. Sorlin, V. Stoica, B. Streicher, J. T. Taylor, O. Teng- blad, S. Terashima, Y. Togano, E. Uberseder, J. Van de Walle, P. Velho, V. Volkov, A. Wagner, F. Wamers, H. Weick, M. Weigand, C. Wheldon, G. Wilson, C. Wimmer, J. S. Winfield, P. Woods, D. Yakorev, M. V. Zhukov, A. Zilges, and K. Zuber (R3B Collaboration), "Systematic investigation of projectile fragmentation using beams of unstable B and C isotopes," Phys. Rev. C 93, 054601 (2016).

[57] J. Okolowicz, Y.H. Lam, M. Ploszajczak, A.O. Macchiavelli, and N.A. Smirnova, "Consistent analysis of one-nucleon spectroscopic factors involving weakly- and strongly-bound nucleons," Phys. Lett. B 757, 303 - 306 (2016).

[58] L. V. Grigorenko, I. G. Mukha, I. J. Thompson, and M. V. Zhukov, "Two-proton widths of ${ }^{12} \mathrm{O},{ }^{16} \mathrm{Ne}$, and three-body mechanism of Thomas-Ehrman shift," Phys. Rev. Lett. 88, 042502 (2002).

[59] J. Okołowicz, M. Płoszajczak, and W. Nazarewicz, "On the origin of nuclear clustering," Prog. Theor. Phys. Supp. 196, 230-243 (2012).

[60] J. Okołowicz, W. Nazarewicz, and M. Płoszajczak, "Towards understanding the microscopic origin of nuclear clustering," Fortschr. Phys. 61, 66 (2013).

[61] J. Okołowicz, N. Michel, W. Nazarewicz, and M. Płoszajczak, "Asymptotic normalization coefficients and continuum coupling in mirror nuclei," Phys. Rev. C 85, 064320 (2012).

[62] J. Okołowicz and M. Płoszajczak, "Exceptional points in the scattering continuum," Phys. Rev. C 80, 034619 (2009).

[63] J. Okołowicz, M. Płoszajczak, and I. Rotter, "Dynamics of quantum systems embedded in a continuum," Phys. Rep. 374, 271 (2003).

[64] M. Płoszajczak and J. Okołowicz, personal communication (2020).

[65] N. Michel, W. Nazarewicz, and M. Płoszajczak, "Threshold effects in multichannel coupling and spectroscopic factors in exotic nuclei," Phys. Rev. C 75, 031301(R) (2007).

[66] X. Mao, J. Rotureau, W. Nazarewicz, N. Michel, R. M. Id Betan, and Y. Jaganathen, "Gamow Shell Model description of $\mathrm{Li}$ isotopes and their mirror partners," (2020), arXiv:2004.02981 [nucl-th].

[67] S. Wang and J. Wylie, personal communication (2020).

[68] Tore Berggren, "Expectation value of an operator in a resonant state," Phys. Lett. B 373, 1 - 4 (1996).

[69] N. Michel, W. Nazarewicz, and M. Płoszajczak, "Isospin mixing and the continuum coupling in weakly bound nuclei," Phys. Rev. C 82, 044315 (2010).

[70] W. A. Peters, T. Baumann, B. A. Brown, J. Brown, P. A. DeYoung, J. E. Finck, N. Frank, K. L. Jones, J.L. Lecouey, B. Luther, G. F. Peaslee, W. F. Rogers, A. Schiller, M. Thoennessen, J. A. Tostevin, and K. Yoneda, "Neutron knockout of ${ }^{12} \mathrm{Be}$ populating neutron-unbound states in ${ }^{11}$ Be," Phys. Rev. C 83, 057304 (2011). 